\title{
Free Surface Gravity Flow Due to a Submerged Body in Uniform Current
}

\author{
Y. A. $\mathrm{SEMENOV}{ }^{1}$ ， G. X. $\mathrm{WU}^{1} \dagger$ \\ ${ }^{1}$ Department of Mechanical Engineering, University College London, London WC1E 6BT, UK
}

(Received xx; revised xx; accepted xx)

The hydrodynamic problem of a body submerged beneath a free surface in a current is considered. The mathematical model used is based on the velocity potential theory with fully nonlinear boundary conditions. The integral hodograph method used previously in a simply connected domain is extended for the present problem to a doubly connected domain. Analytical expressions for the complex velocity and for the complex potential are derived in a rectangular region in a parameter plane, involving the Theta-functions. The boundary value problem is transformed into a system of two integral equations for the velocity modulus on the free surface and for the slope of the submerged body surface in the parameter plane, which are solved through the successive approximation method. Case studies are undertaken both for a smooth body and for a hydrofoil with a sharp edge. Results for the free surface shape, pressure distribution as well as resistance and lift are presented for a wide range of Froude numbers and depths of submergence. It further confirms that at each submergence below a critical value there is a range of Froude numbers within which steady solution may not exist. This range increases as the submergence decreases. This applies to both a smooth body and a hydrofoil. At the same time it is found that at any Froude number beyond a critical value the wave amplitude and the resistance decrease as the body approaches the free surface. In these cases nonlinear effects become more pronounced.

\section{Introduction}

The problem of free-surface gravity flow past a submerged body has received extensive attention for more than one century due to its important relevance to wave resistance of a ship. Among the earliest work is that by Lamb, summarized in his book (Lamb (1932)), in which uniform flow past a submerged circular cylinder was modelled by a dipole at the centre of the cylinder, whose strength was the same as that without the free surface. The linearized free surface boundary condition was then satisfied exactly, while the impermeable boundary condition on the body surface was satisfied approximately. The approach was later known as the first approximation. Havelock (1927) introduced a correction to the first approximation by taking images alternately regarding the body surface and the free surface. Then, Havelock (1936) obtained a solution for the circular cylinder in the form of infinite series, in which each term satisfied the linearized free surface boundary condition. The unknown coefficients in the series were obtained through the body surface boundary condition. In such a way, both the linearized free surface and body surface boundary conditions were satisfied exactly. The latter would be an approximation only in the sense that the infinite series was truncated for practical calculation. From the solution, the results for both the wave resistance and the vertical force, or the lift were obtained.

$\dagger$ Email address for correspondence: g.wu@ucl.ac.uk 
Wehausen \& Liatone (1960) solved the problem for a circular cylinder by using the formulae of Kochin (1937) and Milne-Thomson (1968), alternatively, and also provided an outstanding and extended review of earlier work on this problem. Later Wehausen (1973) further gave a comprehensive review specifically on wave resistance of a ship. The analytical solutions for three dimensional problems include those for a sphere in infinite water depth (Wu \& Eatock Taylor (1988)), in finite water depth (Wu (1995)) and in a channel (Wu (1998)).

The earlier studies mentioned above assumed that disturbances on the free surface were small. This might be justified when the body was deeply submerged or the body was elongated in the incoming stream direction. In order to account for nonlinear effects on the free surface, Tuck (1965) developed a consistent second-order wave theory, in which the nonlinear free surface boundary conditions on the unknown position was expanded to the mean level of the water surface and terms of up to second order were kept. He demonstrated through the second-order correction that the nonlinearity of the freesurface condition was important for the wave resistance, even when the ratio of the body submergence to its radius is relatively large, for example at 4.

The non-linear free-surface effects were also investigated by Salvesen (1969) using higher-order perturbation theory. He derived a consistent second-order solution and attempted to include the third order effect for the free surface elevation at far field. The solution procedure was not limited to a circular cylinder and results were obtained for hydrofoils of finite thickness. Based on the results from the calculations, as well as comparisons with experiments, he confirmed the conclusion of Tuck (1965), that the linearize theory was inadequate, especially when the submergence was small.

Dagan (1971) employed the method of matched asymptotic expansions to analyse the flow past a circular cylinder close to a free surface at high Froude number. The inner solution was based on the non-linear gravity-free flow past a doublet, while the outer solution was that from the linearized free surface boundary condition. His solution became close to the solution with the linearized free surface condition, as the submergence of the cylinder became large.

Free surface flow with obstruction on the bottom of the fluid was studied by Forbes \& Schwartz (1982). Vanden-Broeck (1987) considered a semicircular obstacle while Dias \& Vanden-Broeck (1989) considered a triangular shape using the conformal mapping method coupled with the boundary integral method. By using the Cauchy integral equation formula, Dias \& Vanden-Broeck (2004) derived a system of integral equations for an arbitrarily shaped bottom.

Chapman \& Vanden-Broeck (2006) investigated nonlinear gravity waves over a rough bottom or submerged object at small Froude number using the technique of exponential asymptotics. At the limit of zero Froude number, the dominance of gravity means that the free surface tends to a rigid flat surface.

An approximate solution for a submerged cylinder was also obtained by Kiselev \& Troepol'skaya (1996) assuming that the velocity magnitude at the free surface was approximately constant, which was exact when the Froude number was infinite or the gravity effect was ignored. A related problem is that free surface flow past a source or sink. King \& Bloor (1989) considered the fully nonlinear flow problem. They used a conformal mapping technique and derived, in particular, a nonlinear integro-differential equation in terms of velocity angle on the free surface. However, they did not present detailed numerical results.

The problem of the flow past a submerged hydrofoil with cavity effect in the wake was studied by Faltinsen \& Semenov (2008) with a fully nonlinear boundary condition on the free surface. Because the flow region is a doubly connected domain, they introduced 
a cut along the streamline starting at the closure point of the cavity of the hydrofoil and included boundary conditions on the both sides of the streamline into the formulation of the problem. When the cavity effect is ignored, the streamline used for the cut started from the trailing edge. The integral hodograph method (Semenov \& Iafrati (2006)) could then be used, in which the complex velocity potential of the flow could be written in terms of integrals in a parameter plan. The solution of the integral equations was obtained by a numerical method. For a smooth body, the streamline used for the cut in the method of Faltinsen \& Semenov (2008) would have to start from the stagnation point of the body. However, the position of the stagnation point is not known in advance and it would have to be determined as a part of the solution procedure.

In the linearized free surface problem, it is commonly assumed that for a body in the steady current the free surface flow will eventually become steady. Therefore, the steady solution is usually obtained directly. However, for the nonlinear free surface problem, it was noticed that the steady solution could not always be achieved when the time domain method was used (Haussling \& Coleman (1979)). Scullen \& Tuck (1995) then assumed that the flow was already steady and attempted to find its solution using the iteration method for the nonlinear steady free surface boundary condition. They found that at given a submergence of a circular cylinder they were not able obtain the steady solution within certain range of the Froude number. This range reduced as the submergence increased and disappeared when the submergence was sufficiently large. This was consistent with what was observed by Haussling \& Coleman (1979) using the time domain method. One should note that wave blocking might occur when the local wave upstream meets the current and the total velocity at the crest becomes zero. What has been observed and discussed by Haussling \& Coleman (1979) and Scullen \& Tuck (1995) suggests that no steady solution can be found near the blocking condition.

Scullen \& Tuck (1995) also introduced a vortexes located at the centre of the circular cylinder. Through adjusting the circulation, they confirmed numerically that the wave at the far downstream could virtually disappear and the wave resistance on the cylinder became zero, which had be easily predicted by the linear theory.

Here, we present a fully nonlinear solution to the problem of an arbitrarily shaped body which may be a cylinder or a hydrofoil, moving beneath the free surface, or equivalently a fixed body in a uniform incoming current, in the presence of gravity. In contrast to the method used by Faltinsen \& Semenov (2008) for the doubly connected domain, the cut used in the present formulation does not have to be a streamline which has to intersect the smooth body at the stagnation point. We can use an arbitrary line linking the body and the free surface for the cut. It greatly simplifies the solution and the numerical procedure, and enables features of the flow to be investigated more easily. A further development of this work is that we use the apparatus of elliptic Theta-functions, which enables the problem in a doubly connected domain in the physical plane to be converted into a simply connected domain in the parameter plane. In particular, a rectangle can be used in the latter, instead of the first quadrant commonly adopted previously for simply connected domains (e.g. Semenov \& Wu (2013)). Two of its parallel sides correspond two sides of the cut, on which a periodic condition can be adopted.

Specifically, our solution method follows that proposed by Michell (1890), Joukovskii (1890) for steady jet flows of an ideal fluid. The key step of the method is to find the two governing functions: the complex velocity and the derivative of the complex potential, both defined in an auxiliary parameter region. For the case of a doubly connected flow domain these functions can be effectively obtained using the mathematical apparatus of elliptic Theta-functions.

The problem is formulated in section 2, where the governing functions are derived. For 
determination of the complex velocity, we have derived an integral formula for a mixed boundary-value problem for an analytical function defined in the rectangular auxiliary parameter region. This formula makes it possible to determine the analytical function from values of its modulus and argument given on the horizontal sides of the rectangle. The complex velocity obtained using this formula explicitly includes the variation of the velocity modulus along the free boundary and the variation of the velocity angle along the body as functions of the parameter variable. The system of integral equations for the velocity angle along the body and the velocity magnitude along the free surface are derived by imposing the kinematic and dynamic boundary conditions.

In section 3, a method of successive approximations adopted for solving the integral equations is outlined. The problem without gravity is first considered in section 3.1. As a validation step for problem with gravity, in section 3.2 comparisons are made between the obtained results and those by Scullen \& Tuck (1995). The results are presented for the circular cylinder in terms of the free surface elevation, streamline patterns and force coefficients in a wide range of Froude numbers and depths of submergence. Particular attention is given to small depths of submergence for which nonlinear effects are most pronounced. In section 3.3 the solution procedure is adopted to predict flow past a submerged hydrofoil. Conclusions are given in section 4 .

\section{Theoretical analysis}

We consider a two-dimensional problem of the steady free surface flow of infinite depth past a submerged cylindrical body with characteristic length $L$. Definition sketch is shown in figure 1a. A Cartesian coordinate system $X Y$ is defined with the origin at a point inside the body and the $X$-axis along the velocity direction of the incoming flow with a constant speed $U$. The $Y$-axis points vertically upwards. The fluid is assumed to be inviscid and incompressible, and the flow is irrotational. The velocity potential theory can then be used in such a case. The body is assumed to have an arbitrary shape which can be defined by the slope of the body as a function of the arc length coordinate $S$, or $\beta_{b}=\beta_{b}(S)$. The free surface is defined by the function $Y(X)$. In general, the free surface at far upstream remains flat, while there will be waves at far downstream. The solution which we are seeking therefore has the limit $Y(X)_{X \rightarrow-\infty}=H$, where $H$ is submergence of the body, measured from the origin of the coordinate system. The condition at far downstream, or $X \rightarrow \infty$ will be discussed when the solution method is presented.

We will solve the problem through determining the complex potential of the flow, $W(Z)$ with $Z=X+i Y$. For the steady flow, the kinematic conditions on the body surface and the free surface mean that the stream function is constant, or $\Im[W(Z)]=$ const., as they are both streamlines. However, the constant on the body surface may be different from that on the free surface. The dynamic boundary condition on the free surface is obtained from the Bernoulli equation

$$
\rho \frac{V^{2}}{2}+\rho g Y=\rho \frac{U^{2}}{2}+\rho g H
$$

where $U$ is the speed of incoming stream, $\rho$ is the liquid density, $V=|d W / d Z|$ is the magnitude of the velocity, $g$ is gravity acceleration. Two different Froude numbers can be defined based on the characteristic length $L$ or the depth of submergence $H$, respectively

$$
F=\frac{U}{\sqrt{g L}}, \quad F_{h}=\frac{U}{\sqrt{g H}} .
$$

Using non-dimensionalization based on $U, L, \rho$, we have $v=V / U, x=X / L, y=Y / L$, 
a)

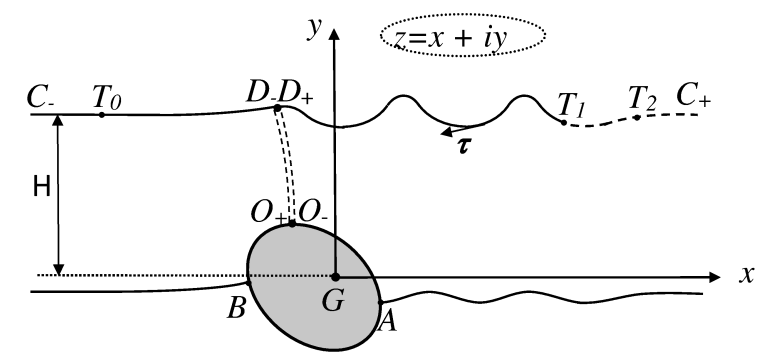

b)

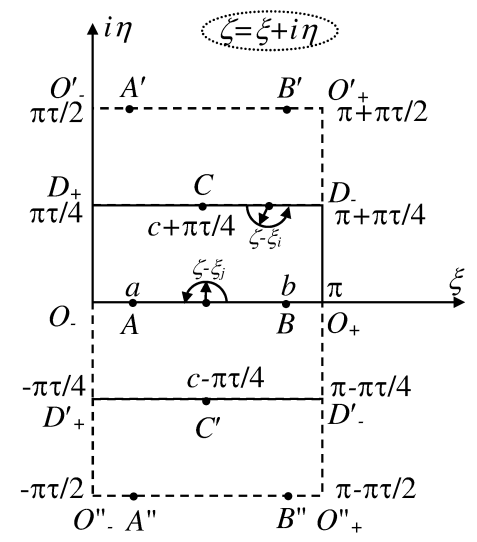

Figure 1. (a) Physical plane and (b) parameter $\zeta$ - plane.

$h=H / L$ and $W(Z)=U L w(z)$. Equation $(2.1)$ takes the form

$$
v^{2}=1-\frac{2(y-h)}{F^{2}} .
$$

Equation (2.3) gives the velocity magnitude along the free surface as a function of wave elevation $y$ which is a function of $x$. When the gravity effect is ignored, or $F \rightarrow \infty$, the velocity magnitude on the free surface is a constant.

\subsection{General approach based on the hodograph method.}

Usually, finding the function $w=w(z)$ directly is a complicated problem. Instead, Michell (1890) and Joukovskii (1890) proposed to introduce an auxiliary parameter plane, or $\zeta$-plane, which was typically chosen as the upper half-plane. Then, they considered two functions, which were the complex potential $w$ and the function $\omega=$ $-\ln (d w / d z)$ in the parameter plane. When $w(\zeta)$ and $\omega(\zeta)$ are obtained as function of $\zeta$, the velocity field and the function mapping the parameter plane onto the physical plane can be determined as follows:

$$
\frac{d w}{d z}=\exp [-\omega(\zeta)], \quad z(\zeta)=z_{0}+\int_{0}^{\zeta} \frac{d w}{d z} / \frac{d w}{d \zeta} d \zeta
$$

where $z_{0}=z(0)$ in the physical plane corresponds to the origin in the $\zeta$-plane.

The flow region beneath the free surface and outside the body is a doubly connected domain. A canonical region of a doubly connected domain is an annulus. By making a cut connecting the external and the internal circles of the annulus, the double connected region becomes simply connected. As shown in figure $1 a, O_{-} D_{+}$and $O_{+} D_{-}$are the two sides of the cut which could have an arbitrary shape, but form a right angle with the flow boundary at both the body surface and the free surface. The simply connected flow region including $O_{-} D_{+}$and $O_{+} D_{-}$is then transformed into the domain bounded by a rectangle $\mathrm{O}_{-} \mathrm{O}_{+} \mathrm{D}_{-} \mathrm{D}_{+}$in the parameter plane. We choose the rectangle as the parameter domain to provide conformal mapping at points $O_{-}, O_{+}, D_{-}, D_{+}$. Indeed, the rectangle has the right angle at these points, same as that at the corresponding points in the physical plane. For other forms of the parameter region, for which angles at points $O_{-}, O_{+}, D_{-}$, $D_{+}$are different from the right angle, a singularity will appear, which makes it more difficult to find the solution. We may choose the coordinates of the rectangle vertexes 
$O_{-}, O_{+}, D_{-}, D_{+}$as $(0,0),(\pi, 0),(\pi, \pi \tau / 4)$ and $(0, \pi \tau / 4)$, respectively, in the parameter plane shown in figure $1 b$. Here, $\tau$ is an imaginary number. The horizontal length of the rectangle is then equal to $\pi$ and its vertical length is equal to $\pi|\tau| / 4$.

In the flow region, there are two stagnation points marked as $A$, where two streamlines merge into one, and $B$, where a streamline splits into two branches. Positions of these points in parameter plane $\zeta, \zeta=a, \zeta=b$ as well as the position of point $C, \zeta=c+\pi \tau / 4$, corresponding to infinity in the physical plane, should be determined from additional conditions. The interval $0 \leqslant \xi \leqslant \pi$ on the real axis corresponds to the body boundary. The interval $c<\xi \leqslant \pi, \eta=\pi \tau / 4$ corresponds to part of the free surface $D_{-} C_{-}$, and the interval $0 \leqslant \xi<c, \eta=\pi \tau / 4$, corresponds to the other part of the free surface $D_{+} C_{+}$.

It should be noticed that points $C_{-}$as $x \rightarrow-\infty$ and $C_{+}$as $x \rightarrow+\infty$ have been transformed to the same point $C$ in the parameter plane. As discussed in the first paragraph of this section, $y \rightarrow h$ at $C_{-}$while it is a wavy function at $C_{+}$. Thus some treatments are need to ensure they can become the same point in the parameter plane. For this reason in the region between points $T_{1}$ and $T_{2}$ in figure $1 a$, an artificial curved plate is placed on the free surface, which applies a pressure on the surface. The logic of this method is that for steady free surface flow past a body in infinite water depth, the body does not create wave at far upstream but it does at far downstream. If the artificial body is put sufficiently behind the physical body, it does not affect flow near the physical body significantly, as it does not generate wave there. On the other hand, when its shape is adjusted properly, the wave generated by its presence can cancel the wave generated by the physical body at far downstream, leading to a flat surface as $x \rightarrow \infty$. This is similar to what is used by Faltinsen \& Semenov (2008). In Vanden-Broeck (2010) the fact that the free surface is infinite is reflected in an integral derived, where the integration limit tends to infinity. The limit is then truncated, which is equivalent to treating the free surface at far downstream as a flat surface. To adopt this technique here, we can also choose the the artificial body as a flat plate. However, the plate has to be put farther away than a curved body which changes the free surface gradually rather than abruptly.

The flux of wave energy at downstream is related to the force applied to the fluid (Wehausen \& Liatone (1960), Newman (1977)). If the total force due to the submerged body and the artificial plate is zero, then there will be no wave at $x \rightarrow \infty$. As a result $C_{+}$ and $C_{-}$in the physical plane can be transformed to the same point $C$ in the parameter plane. The shape of the curved plate will be the part of the solution. When it is applied, the velocity magnitude $v$ along $T_{1} T_{2}$ gradually tends to 1 towards $x \rightarrow x_{T 2}$, and remains to be 1 further beyond, or $x_{T 2} \leqslant x<\infty$. We should notice that the effect of the artificial plate on the flow near the real body will diminish as $x_{T 1}$ increases. This is because the plate will not generate waves at its far upstream.

When solving free boundary problems, a shape of the auxiliary parameter region is usually chosen with the aim to obtain the solution of a problem in the simplest form with minimal numbers of singular points at which the transformation of the parameter region onto the complex potential $w$ and the function $d w / d z$ regions is not conformal. In the case of simply connected domain, the fluid region is commonly converted to the first quadrant of the parameter plane or half-plane (e.g. Gurevich (1965)). In the case of the double connected flow region here, the additional corner points appear at the intersections of two sides of the cut and the flow boundary. To correspond these corner points, it is more convenient to choose the parameter plane in the form of a rectangle. On the other hand, when the integral equation is constructed in the half-plane or the first quadrant, the polynomial functions are usually used. Here, for the rectangular domain, the polynomial functions will be replaced by Jacobi's Theta-functions (Abramowitz \& Stegun (1964), which are quasi-doubly-periodic functions. Jacobi's functions have been used for solving 
free surface problems involving double connected flow regions, for example by Birkhoff \& Zarantonello (1957), Gurevich (1965) and Terentiev et al. (2011).

\subsection{Expression for complex velocity, $d w / d z$, defined in the $\zeta-$ plane.}

At this stage we denote the angle of the velocity direction along the body as $\beta_{b}(\xi)$ and the velocity magnitude on the free surface as $v(\xi)$. With these notations, we have the following boundary-value problem for the function of complex velocity, $d w / d z$ :

$$
\begin{aligned}
& \left|\frac{d w}{d z}\right|=v(\xi), \quad 0 \leqslant \xi \leqslant \pi, \quad \eta=\pi \tau / 4 . \\
& \chi(\xi)=\arg \left(\frac{d w}{d z}\right)=\left\{\begin{array}{lcc}
-\beta_{b}(\xi), & 0 \leqslant \xi<a, & \eta=0, \\
-\beta_{b}(\xi)-\pi, & a<\xi<b, & \eta=0, \\
-\beta_{b}(\xi)-2 \pi, & b<\xi \leqslant \pi, & \eta=0 .
\end{array}\right. \\
& \frac{d w}{d z}(\xi=0, i \eta)=\frac{d w}{d z}(\xi=\pi, i \eta), \quad 0 \leqslant i \eta \leqslant \pi \tau / 4 .
\end{aligned}
$$

In (2.6) the argument of complex velocity has the jumps equal to $-\pi$ at stagnation points $A(\zeta=a)$ and $B(\zeta=b)$ due to the jump of the velocity direction when passing through the stagnation point. The two vertical sides of the rectangle in the parameter plane correspond to the two sides of the cut in the physical plane. The velocities on both sides of the cut are the same and therefore the condition of periodicity can be applied on the vertical sides the rectangle.

The problem is to find the function $d w / d z$ in the parameter domain, which satisfies the given boundary conditions $(2.5)-(2.7)$. Based on the Cauchy integral, it is possible to derive an expression for $d w / d z$ based on an integral along the boundary involving only given condition. Here we may follow a different approach. With the aim of applying the special points method (Gurevich (1965)), we decompose the body surface $O_{-} A B O_{+}$ in the parameter plane into $N$ intervals $E_{j-1}, E_{j}$, with $j=1, \ldots, N$, and assume that the argument of the velocity is piecewise constant, or $\chi(\xi) \equiv \chi_{j-1}$ for $\xi \in\left(\xi_{j-1} \xi_{j}\right)$, so that the velocity argument changes stepwise from $\chi_{j-1}$ to $\chi_{j}$ at vertex $E_{j}$. Here, $\xi_{0}=0$ and $\xi_{N}=\pi$ are defined. The order of singularity of the function $d w / d z$ at point $E_{j}$ can be determined by analysing the behaviour of its argument when we are moving along an infinitesimal semicircle centred at $\xi=\xi_{j}$ in counter clockwise direction as shown in figure $1 b$. In such a way, the argument of $\left(\zeta-\xi_{j}\right)$ changes by $\pi$, while the corresponding change in the argument of $d w / d z$ is $-\left(\chi_{j}-\chi_{j-1}\right)=-\Delta \chi_{j}$. The Theta-function $\vartheta_{1}(\zeta)$ (see Appendix) to be used to derive $d w / d z$ has only one simple zero at point $\zeta=0$ in the rectangle $O_{-} O_{+} D_{-} D_{+}$(Whittaker \& Watson $(1927)$ ), or $\vartheta_{1}(\zeta) \sim \zeta$ near $\zeta=0$. The argument of function $\vartheta_{1}\left(\zeta-\xi_{j}\right)$ near point $\zeta=\xi_{j}$, $\arg \left[\vartheta_{1}\left(\zeta-\zeta_{j}\right)\right]$, behaves in the same way $\operatorname{as} \arg \left(\zeta-\xi_{j}\right)$. Therefore, near $\zeta=\xi_{j}$ if we write

$$
\frac{d w}{d z} \sim\left[\vartheta_{1}\left(\zeta-\xi_{j}\right)\right]^{-\frac{\Delta \chi_{j}}{\pi}}, \quad j=1, \ldots, N
$$

then the change of the argument of $d w / d z$ is $-\Delta \chi_{j}$. Here (2.8) can be either zero or singular, depending on the sign of the exponent. To take into account the change of $d w / d z$ over each $\xi_{j}$, we may write

$$
\frac{d w}{d z} \sim \prod_{j=1}^{N}\left[\vartheta_{1}\left(\zeta-\xi_{j}\right)\right]^{-\frac{\Delta \chi_{j}}{\pi}}, \quad \Delta \chi=\chi_{j}-\chi_{j-1}, \quad j=1, \ldots N,
$$

when $z(\zeta)$ moves along the body surface. We also decompose the free surface $C_{-} D_{-} D_{+} C_{+}$ 
into $M$ intervals $F_{i-1} F_{i}$, with $i=1, \ldots, M$, and assume that the velocity modulus within each interval $\left(\tilde{\xi}_{j-1}, \tilde{\xi}_{j}\right)$ is constant, $v(\tilde{\xi}) \equiv v_{i}$. Similarly the velocity modulus changes stepwise from $v_{i-1}$ to $v_{i}$ at point $F_{i}$. Here $\tilde{\xi}_{0}=\pi$ and $\tilde{\xi}_{M}=0$ are defined. On the basis of the above consideration, we consider the properties of the function $(d w / d z)^{i}=$ $\exp [-i \omega(\zeta))]=\exp (\theta+i \ln v)$, where $\theta=-\arg (d w / d z)$, and $\omega(\zeta)$ is defined in (2.4). The argument of this function is the logarithm of the velocity modulus, and its magnitude is the exponent of the velocity argument. Thus, moving along an infinitesimal semicircle in the $\zeta$-plane centred at $\zeta_{i}=\tilde{\xi}_{i}+\pi \tau / 4$ in counter clockwise direction, $\arg \left[\zeta-\left(\tilde{\xi}_{i}+\pi \tau / 4\right)\right]$ changes by $\pi$, while the corresponding change in the argument of the function $(d w / d z)^{i}$ is $-\left(\ln v_{i}-\ln v_{i-1}\right)$. Therefore, the function behaves near point $\zeta_{i}=\xi_{i}+\pi \tau / 4$ as

$$
\left(\frac{d w}{d z}\right)^{i} \sim\left[\vartheta_{1}\left(\zeta-\xi_{i}-\pi \tau / 4\right)\right]^{-\frac{1}{\pi} \ln \frac{v_{i}}{v_{i-1}}}, \quad i=1, \ldots, M .
$$

Similar to (2.9), we may then write

$$
\frac{d w}{d z} \sim \prod_{i=1}^{M}\left[\vartheta_{1}\left(\zeta-\xi_{i}-\pi \tau / 4\right)\right]^{\frac{i}{\pi} \ln \frac{v_{i}}{v_{i-1}}}, \quad i=1, \ldots, M .
$$

We now construct the expression for the complex velocity based on equations (2.9) and (2.11). Taking into account the requirement in (2.5) to (2.7), we may consider the following function

$$
f(\zeta)=\prod_{j=1}^{N}\left(\frac{\vartheta_{1}\left(\zeta-\xi_{j}\right)}{\vartheta_{1}\left(\zeta-\xi_{j}-\pi \tau / 2\right)}\right)^{-\frac{\Delta \chi_{j}}{\pi}} \prod_{i=1}^{M}\left(\frac{\vartheta_{1}\left(\zeta-\tilde{\xi}_{i}-\pi \tau / 4\right)}{\vartheta_{1}\left(\zeta-\tilde{\xi}_{i}+\pi \tau / 4\right)}\right)^{\frac{i}{\pi} \frac{v_{i}}{v_{i-1}}}
$$

The denominator has no zeros or singularities in the rectangle $O_{-} O_{+} D_{-} D_{+}$and its boundary, and therefore the zeros and singularities are those on the numerator, at $\zeta=\xi_{j}$, $j=1, \ldots, N$, and $\zeta=\tilde{\xi}_{i}+\pi \tau / 4, i=1, \ldots, M$, respectively.

As $\vartheta_{1}(\zeta+\pi)=-\vartheta_{1}(\zeta)$ (see Appendix), equation (2.7) is satisfied. We let $\zeta=\tilde{\xi}+\pi \tau / 4$ on the free surface. Noticing that the nominator and denominator of the first product in (2.12) are complex conjugate as $\tau$ is an imaginary number, we have

$$
\left|\frac{\vartheta_{1}\left(\tilde{\xi}-\xi_{j}+\pi \tau / 4\right)}{\vartheta_{1}\left(\tilde{\xi}-\xi_{j}-\pi \tau / 4\right)}\right|=1 .
$$

Similarly, for point $\zeta=\tilde{\xi}+\pi \tau / 4$ on the line $D_{-} D_{+}$, which is the mirror image of the point $\zeta=\tilde{\xi}-\pi \tau / 4$ about $O_{-} O_{+}$(see figure $1 b$ ), the second product in $(2.12)$ can be evaluated as

$$
\begin{array}{r}
v_{0} \prod_{i=1}^{M} \mid\left(\frac{\vartheta_{1}\left(\tilde{\xi}-\tilde{\xi}_{i}\right)}{\vartheta_{1}\left(\tilde{\xi}-\tilde{\xi}_{i}+\pi \tau / 2\right)}\right)^{\frac{i}{\pi} \ln \frac{v_{i}}{v_{i-1}} \mid=v_{0} \exp \left\{\sum_{1}^{M} \ln \frac{v_{i}}{v_{i-1}} H\left(\tilde{\xi}_{i}-\tilde{\xi}\right)\right\}}= \\
v_{0} \exp \left\{\sum_{1}^{k} \ln \frac{v_{i}}{v_{i-1}}\right\}=v_{k},
\end{array}
$$

where $H\left(\tilde{\xi}_{i}-\tilde{\xi}\right)$ is the Heaviside step function. We can then verify that boundary conditions in (2.5) and (2.6) are satisfied by $f(\zeta)$ apart from a constant. Thus the complex 
velocity may be written as

$$
\frac{d w}{d z}=C \prod_{j=1}^{N}\left(\frac{\vartheta_{1}\left(\zeta-\xi_{j}\right)}{\vartheta_{1}\left(\zeta-\xi_{j}-\pi \tau / 2\right)}\right)^{-\frac{\Delta \chi_{j}}{\pi}} \prod_{i=1}^{M}\left(\frac{\vartheta_{1}\left(\zeta-\tilde{\xi}_{i}-\pi \tau / 4\right)}{\vartheta_{1}\left(\zeta-\tilde{\xi}_{i}+\pi \tau / 4\right)}\right)^{\frac{i}{\pi} \frac{v_{i}}{v_{i-1}}}
$$

in which $C$ is a complex constant.

The products in (2.13) can be recast in the exponential form. We have

$$
\begin{aligned}
\frac{d w}{d z} & =C \exp \left[-\frac{1}{\pi} \sum_{1}^{N} \Delta \chi_{j} \ln \frac{\vartheta_{1}\left(\zeta-\xi_{j}\right)}{\vartheta_{1}\left(\zeta-\xi_{j}-\pi \tau / 2\right)}\right. \\
& \left.+\frac{i}{\pi} \sum_{1}^{M} \Delta \ln v_{i} \ln \frac{\vartheta_{1}(\zeta-\tilde{\xi}-\pi \tau / 4)}{\vartheta_{1}(\zeta-\tilde{\xi}+\pi \tau / 4)}+i \chi(\pi)\right] .
\end{aligned}
$$

By letting $\Delta \chi_{j}=(d \chi / d \xi) / \Delta \xi_{j}$ and $\Delta \ln v_{i}=\ln \left(v_{i} / v_{i-1}\right)=(d \ln v / d \xi) \Delta \xi_{i}$, and taking the limit as the step $\Delta \xi$ in the argument and modulus of velocity tends to zero and, correspondingly, $N \rightarrow \infty$ and $M \rightarrow \infty$, we obtain the integral formula

$$
\begin{aligned}
\frac{d w}{d z} & =v(\pi) \exp \left[-\frac{1}{\pi} \int_{0}^{\pi} \frac{d \chi}{d \xi} \ln \left(\frac{\vartheta_{1}(\zeta-\xi)}{\vartheta_{1}(\zeta-\xi-\pi \tau / 2)}\right) d \xi\right. \\
& \left.+\frac{i}{\pi} \int_{\pi}^{0} \frac{d \ln v}{d \xi} \ln \left(\frac{\vartheta_{1}(\zeta-\xi-\pi \tau / 4)}{\vartheta_{1}(\zeta-\xi+\pi \tau / 4)}\right) d \xi+i \chi(\pi)\right]
\end{aligned}
$$

which gives an expression for the mixed boundary-value problem $(2.5)-(2.7)$ in the rectangle $O_{-} O_{+} D_{-} D_{+}$based on the argument, $\arg [d w / d z]_{\zeta=\xi, \eta=0}=\chi(\xi), 0 \leqslant \xi \leqslant \pi$, $\eta=0$, and the modulus, $|d w / d z|_{\zeta=\xi, \eta=\pi \tau / 4}=v(\xi)$. The important thing to notice here is that the right hand side involves only those boundary values prescribed, either modulus or argument, and not the unknown part. Therefore, $d w / d z$ can be obtained directly once these prescribed values are known. The complex constant $C$ in (2.15) can be determined using the value of the argument of the function $d w / d z$ at point $O_{+}(\zeta=\pi)$ and its modulus at point $D_{-}(\zeta=\pi+\pi \tau / 4)$. We note, that the integral formula (2.15) derived for the complex velocity, $d w / d z$, can be used for solving boundary value problem (2.5) - (2.7) for an arbitrary complex function. By substituting the boundary conditions (2.5) and (2.6) into (2.15) and evaluating the first integral over the step change in the function $\chi(\xi)$ at points $\xi=a$ and $\xi=b$, we obtain the expression for the complex velocity in the rectangle $O_{-} O_{+} D_{-} D_{+}$

$$
\begin{aligned}
\frac{d w}{d z} & =v_{D} \frac{\vartheta_{1}(\zeta-a) \vartheta_{1}(\zeta-b)}{\vartheta_{4}(\zeta-a) \vartheta_{4}(\zeta-b)} \exp \left[\frac{1}{\pi} \int_{0}^{\pi} \frac{d \beta_{b}}{d \xi} \ln \frac{\vartheta_{1}(\zeta-\xi)}{\vartheta_{4}(\zeta-\xi)} d \xi\right. \\
& \left.+\frac{i}{\pi} \int_{\pi}^{0} \frac{d \ln v}{d \xi} \ln \frac{\vartheta_{1}(\zeta-\xi-\pi \tau / 4)}{\vartheta_{4}(\zeta-\xi-\pi \tau / 4)} d \xi-i \beta_{O}\right] .
\end{aligned}
$$

where $\beta_{O_{-}}$is the angle at point $O_{-}$which is zero if point $O_{-}$is the highest point of the body. Here, the relations between Theta-functions $\vartheta_{1}(\xi)$ and $\vartheta_{4}(\xi)$ have been used (see Appendix). The constant $v_{D}$ or the velocity magnitude at point $D_{+}$, is determined by satisfying the velocity at infinity, $\zeta=c+\pi \tau / 4$, which is 1 , as it has been chosen as the reference velocity, or

$$
\left|\frac{d w}{d z}\right|_{\zeta=c+\pi \tau / 4}=1
$$




\subsection{Expression for derivative of the complex potential, $d w / d \zeta$.}

For steady flows, the stream function $\psi=\Im(w)$ takes constant values along the body and the free surface. According to Chaplygin's special point method (Gurevich (1965), Chapter 1(5)), to determine the function $w=w(\zeta)$, it is sufficient to analyse all special points where the mapping is not conformal. These are stagnation points $A(\zeta=a)$ and $B(\zeta=b)$ and point $C(\zeta=c+\pi \tau / 4)$ corresponding to infinity in $w$-plane. The order of $w(\zeta)$ at these points can be determined by analysing the behaviour of the argument of $w(\zeta)$ in the vicinity of these points.

Moving in the counter-clockwise direction around an infinitesimal semicircle centred at the point $\zeta=a, \arg (\zeta-a)$ changes by $\pi$ while the corresponding change of $\arg \left(w-w_{A}\right)$ in the $w$-plane is $2 \pi$. This means that the function $w(\zeta)$ behaves as

$$
w-w_{A} \sim \theta_{1}^{2}(\zeta-a)
$$

near point $A$. Similarly, at the stagnation point $B(\zeta=b)$, the function $w(\zeta)$ behaves as

$$
w-w_{B} \sim \theta_{1}^{2}(\zeta-b) .
$$

Moving in the counter-clockwise direction around an infinitesimal semicircle centred at the point $\zeta=c+\pi \tau / 4, \arg (\zeta-c-\pi \tau / 4)$ increases by $\pi$, while $\arg \left(w-w_{C}\right)$ of the semicircle of an infinite radius linking points $C_{+}$and $C_{-}$in the $w$-plane decreases by $\pi$. Therefore, the function $w(\zeta)$ behaves as

$$
w-w_{C} \sim \theta_{1}^{-1}(\zeta-c-\pi \tau / 4) .
$$

According to symmetry principle, to satisfy the boundary condition $\Im(w)=$ const. on the flow boundary, it is necessary to put special points of the same order at points $A^{\prime}$ $(\zeta=a+\pi \tau / 2)$ and $B^{\prime}(\zeta=b+\pi \tau / 2)$, which are respectively symmetry points of $A$ and $B$ with respect to the side $D_{-} D_{+}$, and at point $C^{\prime}(\zeta=c-\pi \tau / 4)$ which is the symmetry point of $C$ with respect to side $O_{-} O_{+}$.

By differentiating the function $w(\zeta)$, we obtain that the derivative $d w / d \zeta$ which has the following zeros and poles:

$$
\begin{array}{rc}
\theta_{1}(\zeta-a), & \theta_{1}(\zeta-b), \quad \theta_{1}^{-2}(\zeta-c-\pi \tau / 4), \\
\theta_{1}(\zeta-a-\pi \tau / 2), & \theta_{1}(\zeta-b-\pi \tau / 2), \quad \theta_{1}^{-2}(\zeta-c+\pi \tau / 4)
\end{array}
$$

on boundaries of the rectangle and their symmetry lines. Similar to $d w / d z$, we may construct $d w / d \zeta$ in the following form

$$
\frac{d w}{d \zeta}=K^{\prime} e^{-i 2(\zeta-c)} \frac{\vartheta_{1}(\zeta-a) \vartheta_{1}(\zeta-a-\pi \tau / 2) \vartheta_{1}(\zeta-b) \vartheta_{1}(\zeta-b-\pi \tau / 2)}{\vartheta_{1}^{2}(\zeta-c-\pi \tau / 4) \vartheta_{1}^{2}(\zeta-c+\pi \tau / 4)},
$$

where $K^{\prime}$ is a real scale factor. The term $e^{-i 2(\zeta-c)}$ is introduced in the above equation to satisfy kinematic boundary condition on the body and the free surface. In fact, on the free surface, $\zeta=\tilde{\xi}+\pi \tau / 4$, equation (2.18) takes the form

$$
\frac{d w}{d \zeta}=K^{\prime} q^{-\frac{1}{2}} e^{-i 2(\tilde{\xi}-c)} \frac{\vartheta_{1}(\tilde{\xi}-a+\pi \tau / 4) \vartheta_{1}(\tilde{\xi}-a-\pi \tau / 4) \vartheta_{1}(\tilde{\xi}-b+\pi \tau / 4) \vartheta_{1}(\tilde{\xi}-b-\pi \tau / 4)}{\vartheta_{1}^{2}(\tilde{\xi}-c) \vartheta_{1}^{2}(\tilde{\xi}-c+\pi \tau / 2)} .
$$

We notice that $\vartheta_{1}\left(\tilde{\xi}-\tilde{\xi}^{\prime}+\pi \tau / 4\right)$ and $\vartheta_{1}\left(\tilde{\xi}-\tilde{\xi}^{\prime}-\pi \tau / 4\right)$ are complex conjugate to each other and

$$
\vartheta_{1}(\tilde{\xi}-c+\pi \tau / 2)=i q^{-\frac{1}{4}} e^{-i(\tilde{\xi}-c)} \vartheta_{4}(\tilde{\xi}-c) .
$$


Thus,

$$
\Im(d w)=\Im\left[(d w / d \zeta)_{\zeta=\tilde{\xi}+\pi \tau / 4} d \tilde{\xi}\right]=0 .
$$

On the body surface, $\zeta=\xi$, we may use

$$
\vartheta_{1}(\zeta-\pi \tau / 2)=-i q^{\frac{1}{4}} e^{i \zeta} \vartheta_{4}(\zeta)
$$

to write $(2.18)$ as

$$
\frac{d w}{d \zeta}=K e^{i(2 c-a-b)} \frac{\vartheta_{1}(\xi-a) \vartheta_{4}(\xi-a) \vartheta_{1}(\xi-b) \vartheta_{4}(\xi-b)}{\vartheta_{1}^{2}(\xi-c-\pi \tau / 4) \vartheta_{1}^{2}(\xi-c+\pi \tau / 4)},
$$

where $K=K^{\prime} q^{-1}$. The numerator is real following definitions of the functions $\vartheta_{1}(\zeta)$ and $\vartheta_{4}(\zeta)$. The denominator is also real since $\vartheta_{1}^{2}(\xi-c-\pi \tau / 4)$ and $\vartheta_{1}^{2}(\xi-c+\pi \tau / 4)$ are complex conjugate quantities. Thus,

$$
\Im(d w)=\Im\left[(d w / d \zeta)_{\zeta=\xi} d \xi\right]=0,
$$

if $2 c-a-b=0$ which will be shown later. Using this, equation (2.20) becomes

$$
\frac{d w}{d \zeta}=K \frac{\vartheta_{1}(\zeta-a) \vartheta_{4}(\zeta-a) \vartheta_{1}(\zeta-b) \vartheta_{4}(\zeta-b)}{\vartheta_{1}^{2}(\zeta-c-\pi \tau / 4) \vartheta_{1}^{2}(\zeta-c+\pi \tau / 4)} .
$$

Dividing (2.21) by (2.16), we obtain the derivative of the mapping function as

$$
\begin{aligned}
\frac{d z}{d \zeta}= & \frac{K}{v_{D}} \frac{\theta_{4}^{2}(\zeta-a) \theta_{4}^{2}(\zeta-b)}{\theta_{1}^{2}(\zeta-c-\pi \tau / 4) \theta_{1}^{2}(\zeta-c+\pi \tau / 4)} \\
& \times \exp \left[-\frac{1}{\pi} \int_{0}^{\pi} \frac{d \beta_{b}}{d \xi} \ln \frac{\theta_{1}(\zeta-\xi)}{\theta_{4}(\zeta-\xi)} d \xi-\frac{i}{\pi} \int_{\pi}^{0} \frac{d \ln v}{d \xi} \ln \frac{\theta_{1}(\zeta-\xi-\pi \tau / 4)}{\theta_{4}(\zeta-\xi-\pi \tau / 4)} d \xi+i \beta_{O}\right] .
\end{aligned}
$$

whose integration along the intervals $0 \leqslant \xi<c$ and $c<\xi \leqslant \pi$ at $\eta=\pi \tau / 4$ in the $\zeta$-plane provides the parts $D_{+} C_{+}$and $D_{-} C_{-}$of the free surface $C_{-} C_{+}$in $\zeta$-plane, respectively. The parameters $a, b, c, \tau$ and $K$, and the functions $\beta_{b}(\xi)$ and $d(\ln v) / d \xi$ have to be determined from physical considerations and the kinematic boundary condition on the body surface and the dynamic boundary conditions on the free surface.

\subsection{System of equations for parameters $a, b, c, \tau$ and $K$.}

At infinity, point $C_{-} C_{+}\left(\zeta_{C}=c+\pi \tau / 4\right)$, the velocity approaches unit (since this velocity is chosen as the reference velocity) and its direction is along the $x$-axis. Therefore, the argument of the complex velocity (2.16) at point $\zeta_{C}$ should be equal to zero

$$
\arg \left(\frac{d w}{d z}\right)_{\zeta=\zeta_{C}}=1 .
$$

The scale factor $K$ is determined by the length $S_{b}$ which is the perimeter of the body cross-section

$$
\int_{0}^{\pi} \frac{d s_{b}}{d \xi} d \xi=S_{b}
$$

where

$$
\frac{d s_{b}}{d \xi}=\left|\frac{d z}{d \zeta}\right|_{\zeta=\xi}
$$

The free surface on the left and right hand sides at infinity has the same value of $y$-coordinate. This is also equivalent to that the stream function $\psi=\Im(w)$ is continuous across the cut, or $\Im\left(w_{D_{-}}\right)-\Im\left(w_{D_{+}}\right)=0$. By integrating $\Im(d w / d \zeta)$ along $D_{-} D_{+}$passing 
the point $\zeta_{C}$ along a semi-circle $C^{\prime}$ of an infinitesimal radius $\varepsilon$, at which $d w / d \zeta$ in Eq.(2.21) has the second order singularity, we have

$$
\begin{gathered}
\Im\left(\int_{\pi}^{c+\varepsilon} \frac{d w}{d \zeta} d \zeta+\oint_{C^{\prime}} \frac{d w}{d \zeta} d \zeta\right. \\
\left.+\int_{c-\varepsilon}^{0} \frac{d w}{d \zeta} d \zeta\right)=\Im\left(\oint_{C^{\prime}} \frac{d w}{d \zeta} d \zeta\right)=\Im\left(i \pi \operatorname{Res}_{\zeta=\zeta_{C}} \frac{d w}{d \zeta}\right) \\
=\Im\left\{i \pi \frac{d}{d \zeta}\left[\frac{d w}{d \zeta}\left(\zeta-\zeta_{C}\right)^{2}\right]_{\zeta=\zeta_{C}}\right\} .
\end{gathered}
$$

Here the first and third terms on the left hand are zero because $\Im(w)=$ const. on the free surface. From this equation it follows

$$
a+b=2 c .
$$

The depth of submergence, $h$, and the flowrate, $Q$, between the body and the free surface are related. Therefore, instead of a condition for the depth $h$, we can use the following condition for the given flowrate $Q$, which is the integral of the derivative of the complex potential along the side $\mathrm{O}_{-} D_{+}$

$$
\Im\left(\int_{0}^{\pi \tau / 4} \frac{d w}{d \zeta} d \zeta\right)=Q .
$$

We may place a vortex with circulation $\Gamma$ at the centre of the cylinder, which can be nondimensionalized as $\gamma=\Gamma /(2 \pi U L)$. For a circular cylinder, this does not affect the impermeable body surface boundary condition, but does change the positions of the stagnation points and also affects the free surface boundary. For a hydrofoil, $\gamma$ should be determined through the Kutta condition at the trailing edge.

Integrating $d w / d \zeta$ along the body surface in the parameter plane, we have

$$
\Re\left(\int_{0}^{\pi} \frac{d w}{d \zeta} d \zeta\right)=2 \pi \gamma
$$

In the case $\gamma \neq 0$, the real part of the potential, $\phi=\Re(w)$, have a jump on the sides $O_{-} D_{-}$and $O_{+} D_{+}$of the cut, while the complex velocity, $d w / d z$ and the stream function $\psi=\Im(w)$ are still continuous across the cut.

Equations (2.23) - (2.27) allow us to determine the unknown parameters $a, b, c, \tau$ and $K$, which appear in the governing equations $(2.16),(2.21)$ and $(2.22)$, once the functions $v(\xi)$ and $\beta_{b}(\xi)$ are specified.

\subsection{Integro-differential equations for functions $\beta_{b}(\xi)$ and $v(\xi)$.}

\subsubsection{Kinematic boundary condition on the body surface.}

By integrating the modulus of the mapping function (2.22) along the side $O_{-} O_{+}$in the parameter plane, we can obtain the spatial coordinate along the body as a function of the parameter variable

$$
s_{b}(\xi)=\int_{0}^{\xi} \frac{d s_{b}}{d \xi^{\prime}} d \xi^{\prime},
$$

where $d s_{b} / d \xi=|d z / d \zeta|_{\zeta=\xi, \eta=0}$. Since the function $\beta_{b}\left(s_{b}\right)$ is known, the function $\beta_{b}(\xi)$ can be determined from the following integro-differential equation:

$$
\frac{d \beta_{b}}{d \xi}=\frac{d \beta_{b}}{d s_{b}} \frac{d s_{b}}{d \xi} .
$$


By substituting $d z / d \zeta$ from (2.22), this equation takes the form

$$
\begin{aligned}
\frac{d \beta_{b}}{d \xi} & =\kappa\left[s_{b}(\xi)\right] \frac{K}{v_{D}}\left|\frac{\theta_{4}^{2}(\xi-a) \theta_{4}^{2}(\xi-b)}{\theta_{1}^{2}(\xi-c-\pi \tau / 4) \theta_{1}^{2}(\xi-c+\pi \tau / 4)}\right| \\
& \times \exp \left[-\frac{1}{\pi} \int_{0}^{\pi} \frac{d \beta_{b}}{d \xi^{\prime}} \ln \frac{\theta_{1}\left(\xi-\xi^{\prime}\right)}{\theta_{4}\left(\xi-\xi^{\prime}\right)} d \xi^{\prime}-\frac{i}{\pi} \int_{\pi}^{0} \frac{d \ln v}{d \xi^{\prime}} \ln \frac{\theta_{1}\left(\xi-\xi^{\prime}-\pi \tau / 4\right)}{\theta_{4}\left(\xi-\xi^{\prime}-\pi \tau / 4\right)} d \xi^{\prime}\right],
\end{aligned}
$$

where $\kappa\left(s_{b}\right)=d \beta_{b} / d s_{b}$ is the curvature of the body.

\subsubsection{Nonlinear boundary condition on the free surface: integral equation for the} function $d \ln v / d \xi$.

The velocity magnitude on the free surface can be obtained from Bernoulli equation (2.3) in iteration manner using the initial guess $y(\xi)=h$ and

$$
v(\xi)=\sqrt{1-\frac{2(y(\xi)-h}{F^{2}}} .
$$

Then, at each branch of the free surface, $C_{-} D_{-}(c<\xi \leqslant \pi)$ and $C_{+} D_{+}(0 \leqslant \xi<c)$, its elevation can be obtained from

$$
y(\xi)_{\{c<\xi \leqslant \pi, 0 \leqslant \xi<c\}}=y_{D}+\Im\left(\left.\int_{\{\pi, 0\}}^{\xi} \frac{d z}{d \zeta}\right|_{\zeta=\xi+\pi \tau / 4} d \xi\right),
$$

where the integrand can be obtained from $(2.22)$, and $y_{D}$ is the vertical coordinate of point $D$ and can be obtained from

$$
y_{D}=\Im\left(\left.i \int_{0}^{\pi|\tau| / 4} \frac{d z}{d \zeta}\right|_{\zeta=i \eta} d \eta\right) .
$$

This iteration converges well for the part $C_{-} D_{-}$of the free surface for which $y(\xi)$ changes monotonically, if $D$ is chosen appropriately. For the wavy part $C_{+} D_{+}$the convergence of iteration is more difficult to achieve, which requires to apply more sophisticated approach.

Differentiating equation (2.3) with respect to the arc length coordinate along the free surface and taking into account that slope of the free surface $\delta=\arcsin (d y / d s)=\pi+\beta$ is angle between unit tangential vector $\tau$ and the $x$-axis, we obtain

$$
F^{2} v^{2} \frac{d \ln v}{d s}-\sin \beta=0,
$$

where the velocity direction $\beta$ can be determined from (2.16) by putting $\zeta=\xi+\pi \tau / 4$ :

$$
\begin{aligned}
\beta(\xi) & =\beta_{O}-\Im\left\{\ln \frac{\vartheta_{1}(\xi-a+\pi \tau / 4) \vartheta_{1}(\xi-b+\pi \tau / 4)}{\vartheta_{4}(\xi-a+\pi \tau / 4) \vartheta_{4}(\xi-b+\pi \tau / 4)}\right. \\
& \left.-\frac{1}{\pi} \int_{0}^{\pi} \frac{d \beta_{b}}{d \xi^{\prime}} \Im\left[\ln \frac{\vartheta_{1}\left(\xi-\xi^{\prime}+\pi \tau / 4\right)}{\vartheta_{4}\left(\xi-\xi^{\prime}+\pi \tau / 4\right)}\right] d \xi^{\prime}-\frac{1}{\pi} \int_{\pi}^{0} \frac{d \ln v}{d \xi^{\prime}} \ln \left|\frac{\vartheta_{1}\left(\xi-\xi^{\prime}\right)}{\vartheta_{4}\left(\xi-\xi^{\prime}\right)}\right| d \xi^{\prime}\right\} .
\end{aligned}
$$

Using the relationships

$$
\frac{d v}{d s}=v \frac{d \ln v}{d \xi} / \frac{d s}{d \xi}
$$

and

$$
\frac{d s}{d \xi}=\left|\frac{d z}{d \zeta}\right|_{\zeta=\xi+\pi \tau / 4}=\frac{1}{v(\xi)}\left|\frac{d w}{d \zeta}\right|_{\zeta=\xi+\pi \tau / 4}=\frac{K}{v(\xi)}\left|\frac{\theta_{4}^{2}(\xi-a+\pi \tau / 4) \theta_{4}^{2}(\xi-b+\pi \tau / 4)}{\theta_{1}^{2}(\xi-c) \theta_{4}^{2}(\xi-c)}\right| .
$$


We can rewrite (2.34) with the following integral equation in terms of the function $d \ln v / d \xi$ over the interval $0 \leqslant \xi<c$ :

$$
F^{2} v^{3} \frac{d \ln v}{d \xi}+P_{1}(\xi) \sin \left[\frac{1}{\pi} \int_{\pi}^{0} \frac{d \ln v}{d \xi^{\prime}} \ln \left|\frac{\theta_{1}\left(\xi-\xi^{\prime}\right)}{\theta_{4}\left(\xi-\xi^{\prime}\right)}\right| d \xi^{\prime}+P_{2}(\xi)\right]=0
$$

where

$$
\begin{gathered}
v(\xi)=v_{D} \exp \left(\int_{0}^{\xi} \frac{d \ln v}{d \xi^{\prime}} d \xi^{\prime}\right), \quad P_{1}(\xi)=K\left|\frac{\theta_{4}^{2}(\xi-a+\pi \tau / 4) \theta_{4}^{2}(\xi-b+\pi \tau / 4)}{\theta_{1}^{2}(\xi-c) \theta_{4}^{2}(\xi-c)}\right|, \\
P_{2}(\xi)=\Im\left\{\ln \frac{\theta_{1}(\xi-a+\pi \tau / 4) \theta_{1}(\xi-b+\pi \tau / 4)}{\theta_{4}(\xi-a+\pi \tau / 4) \theta_{4}(\xi-b+\pi \tau / 4)}\right\} \\
+\frac{1}{\pi} \int_{0}^{\pi} \frac{d \beta_{b}}{d \xi^{\prime}} \Im\left\{\ln \frac{\theta_{1}\left(\xi-\xi^{\prime}+\pi \tau / 4\right)}{\theta_{4}\left(\xi-\xi^{\prime}+\pi \tau / 4\right)}\right\} d \xi^{\prime}-\beta_{O} .
\end{gathered}
$$

The velocity modulus function $v(\xi)$ is determined by (2.31) for $c<\xi \leqslant \pi$ and by $(2.38)$ for $0 \leqslant \xi<c$.

\subsection{Numerical approach.}

Discretization of the body and the free surfaces in the $\zeta$-plane. In the discrete form, the solution is sought on a fixed set of points $\xi_{j}, j=1, \ldots, N$ distributed along the side $O_{-} O_{+}, 0 \leqslant \xi_{j} \leqslant \pi, \eta=0$, and fixed set of points $\tilde{\xi}_{i}$ distributed along the intervals $c+\varepsilon \leqslant \tilde{\xi}_{i} \leqslant \pi, i=1, \ldots, M_{1}$ and $0 \leqslant \tilde{\xi}_{i} \leqslant c-\varepsilon, i=M_{1}+1, \ldots, M$ on the side $D_{-} D_{+}$, $\eta=\pi \tau / 4$. These intervals correspond to parts $D_{-} C_{-}$and $D_{+} C_{+}$of the free surface $C_{-} C_{+}$.

The value $\varepsilon$ is chosen to provide the length of the free surface $C_{+} D_{+}$such that $x_{T 1}>$ $5 \lambda$, where $\lambda=2 \pi F^{2}$ is the wave length predicted by a linear theory. The number of nodes on the body and the free surface are chosen in the range $N=100-300$ and $M=500-1000$, respectively, based on the requirement of convergence and accuracy of the solution. For all calculated examples, the difference between the results from the above ranges of $N$ and $M$ starts form the fourth figure only. The points $\xi_{j}$ are distributed in a way to provide a higher density of the points $s_{j}=s_{b}\left(\xi_{j}\right)$ near stagnation points $A(\zeta=a)$ and $B(\zeta=b)$. We have $\xi_{j}=a\left[1-\cos \left(\pi(j-1) /\left(N_{A}-1\right)\right)\right] / 2$, $j=1, \ldots, N_{A}, \xi_{j}=a+(b-a)\left[1-\cos \left(\pi\left(j-N_{A}\right) /\left(N_{B}-N_{A}\right)\right)\right] / 2, j=N_{A}+1, \ldots, N_{B}$ and $\xi_{j}=b+(\pi-b)\left[1-\cos \left(\pi\left(j-N_{B}\right) /\left(N-N_{B}\right)\right)\right] / 2, j=N_{B}+1, \ldots, N$, where $N_{A}=N / 4$ and $N_{B}=3 N / 4$.

The distribution of the points $\tilde{\xi}_{i}$ is chosen similarly to provide a higher density of the points $s_{i}=s\left(\tilde{\xi}_{i}\right)$ on the free boundary closer to the body.

Discrete form of the integro-differential equation for the function $\beta_{b}(\xi)$. The integrals in the equations are evaluated using the linear interpolation of the functions $\beta_{b}(\xi)$ and $\ln v(\tilde{\xi})$ on the segments $\left(\xi_{j-1}, \xi_{j}\right)$ and $\left(\tilde{\xi}_{i-1}, \tilde{\xi}_{i}\right)$, respectively. Then, the integrals in $(2.16)$ are evaluated numerically over each segment using trapezoidal rule.

The method of successive approximations is adopted for solving integro-differential equation (2.30) and integral equation (2.38). In the discrete form equation (2.30) becomes

$$
\frac{\left(\Delta \beta_{b}\right)_{j}^{(k+1)}}{\Delta \xi_{j}}=\frac{\beta_{b}\left[s_{b}^{(k)}\left(\xi_{j}\right)\right]-\beta_{b}\left[s_{b}^{(k)}\left(\xi_{j-1}\right)\right]}{\Delta \xi_{j}}, \quad j=1, \ldots, N,
$$

where the arc length along the body, $s_{b}^{(k)}(\xi)$, is evaluated using $(2.28)$ with $\left(\Delta \beta_{b j} / \Delta \xi_{j}\right)^{(k)}$ 
known at the $(k)^{t h}$ iteration. The iteration process converges very fast. After 5 to 10 iterations the error is below a prescribed tolerance of $10^{-6}$.

Distribution of the velocity magnitude in the transition region $x_{T 1} \leqslant x \leqslant x_{T 2}$. In the $\zeta$-plane, interval $\left(x_{T 1}, x_{T 2}\right)$ corresponds to the interval $\left(\tilde{\xi}_{T 1}, \tilde{\xi}_{T 2}\right)$. The integral equation (2.38) is solved over the interval $0 \leqslant \tilde{\xi}<\tilde{\xi}_{T 1}$ and due to the radiation condition adopted in the present method, velocity magnitude at point $T_{2}$ should be $v\left(x_{T 2}\right)=1$. Therefore, in the transition region, $x_{T 1} \leqslant x \leqslant x_{T 2}$ we use one parameter family for the velocity distribution

$$
v(\bar{x})= \begin{cases}v_{T 1}+\left(1-v_{T 1}\right) \bar{x}+A_{v} \sin (2 \pi \bar{x}) \exp (-\bar{x}), & 0 \leqslant \bar{x} \leqslant 1, \\ 1, & 1<\bar{x}<\infty,\end{cases}
$$

where $\bar{x}=\left(x-x_{T 1}\right) /\left(x_{T 2}-x_{T 1}\right)$ and $A_{v}$ is a parameter. The velocity distribution on the interval $\left(\tilde{\xi}_{T 1}, c\right)$ that is downstream of the point $T_{1}$ does not significantly affect the free surface near the body. This effect will diminish when $x_{T 1}$ is sufficiently large. The parameter $A_{v}$ in (2.40) is chosen to further reduce this effect and obtain zero slope of the free surface at point $x_{M_{1}+1}$, or $\beta\left(\tilde{\xi}_{M_{1}+1}\right)=0$.

The discrete form of the integral equation for the function $d \ln v / d \xi$. In the discrete form equation (2.34) can be written as follows

$$
F^{2}\left(\bar{v}_{i}^{3}\right)^{(k)} \frac{\Delta(\ln v)_{i}^{(k+1)}}{\Delta \phi_{i}}-\beta_{i}^{(k+1)}=\sin \beta_{i}^{(k)}-\beta_{i}^{(k)} .
$$

Due to nonlinearity of $(2.41)$, it is solved through iteration procedure. The system of linear equations to determine unknown $\Delta \ln v_{j}$ at $(k+1)^{t h}$ iteration can be written as follows

$$
a_{i j} \Delta \ln v_{j}=b_{j}, \quad i, j=1, \ldots, n
$$

where $n=M-M_{T}$ is the number of nodes $\tilde{\xi}_{i}$ between points $D_{+}$and $T_{1}, \tilde{\xi}_{M_{T}}=\tilde{\xi}_{T 1}$,

$$
\begin{aligned}
& a_{i j}=A_{i j}+\delta_{i j} F^{2}\left(\bar{v}_{i}^{3}\right)^{(k)} \frac{1}{\Delta \phi_{i}}, \bar{v}_{i}^{(k)}=\frac{v_{i-1}^{(k)}+v_{i}^{(k)}}{2}, \bar{\xi}_{i}=\frac{\tilde{\xi}_{i-1}+\tilde{\xi}_{i}}{2}, \quad \delta_{i j}= \begin{cases}1, & i=j, \\
0, & i \neq j .\end{cases} \\
& A_{i j}=\frac{1}{\pi \Delta \tilde{\xi}_{j}} \int_{\tilde{\xi}_{j-1}}^{\tilde{\xi}_{j}} \ln \left|\frac{\vartheta_{1}\left(\bar{\xi}_{i}-\tilde{\xi}^{\prime}\right)}{\vartheta_{4}\left(\bar{\xi}_{i}-\tilde{\xi}^{\prime}\right)}\right| d \tilde{\xi}^{\prime}, \quad \Delta \phi_{i}=\Re\left(\frac{d w}{d \zeta}\right)_{\zeta=\bar{\xi}_{i}} \Delta \tilde{\xi}_{i} \\
& b_{i}=\sin \beta_{i}^{(k)}+\sum_{1}^{M_{T}} A_{i j} \Delta \ln v_{j}^{(k)}, \quad \beta_{i}^{(k)}=-\sum_{1}^{M} A_{i j} \Delta \ln v_{j}^{(k)}+B_{i}, \\
& B_{i}=\beta_{O_{-}}-\Im\left\{\ln \frac{\vartheta_{1}\left(\bar{\xi}_{i}-a+\pi \tau / 4\right) \vartheta_{1}\left(\bar{\xi}_{i}-b+\pi \tau / 4\right)}{\vartheta_{4}\left(\bar{\xi}_{i}-a+\pi \tau / 4\right) \vartheta_{4}\left(\bar{\xi}_{i}-b+\pi \tau / 4\right)}\right\} \\
& -\sum_{1}^{N} \Delta \beta_{j} \Im\left\{\frac{1}{\pi \Delta \tilde{\xi}_{j}} \int_{\tilde{\xi}_{j-1}}^{\tilde{\xi}_{j}} \ln \left|\frac{\vartheta_{1}\left(\bar{\xi}_{i}-\tilde{\xi}^{\prime}+\pi \tau / 4\right)}{\vartheta_{4}\left(\bar{\xi}_{i}-\tilde{\xi}^{\prime}+\pi \tau / 4\right)}\right|\right\},
\end{aligned}
$$

The iteration procedure is constructed as follows.

(i) Functions $\beta_{b}(\xi)$ and $v(\xi)$ are initialized as $\beta_{b}(\xi)=2 \xi, 0 \leqslant \xi \leqslant \pi$ and $v(\xi) \equiv 1$, together with parameter $A_{v}=0$.

(ii) Equation (2.39) is solved by iteration for a given function $v(\xi)$. For each iteration, equations (2.23) - (2.27) are solved to update $a, b, c, K$ and $\tau$, respectively.

(iii) Apply external iteration to update $v(\xi)$ by solving the system of equations (2.42).

(iv) Return to (ii) and continue until the free surface shape downstream has converged. 

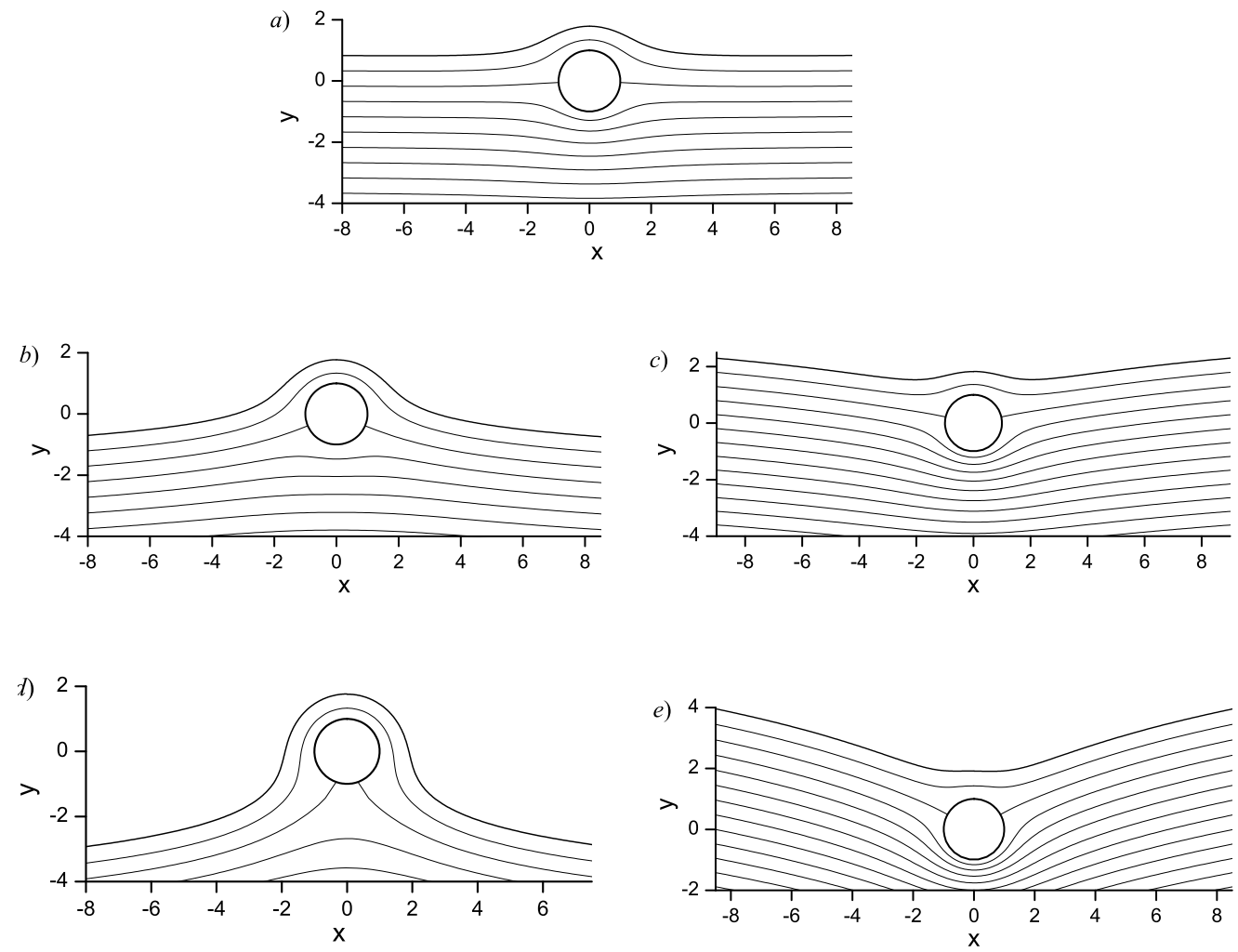

FiguRE 2. Streamline patterns for a submerged circular cylinder at the flowrate $Q=1$ for different circulations: (a) $2 \pi \gamma=0$; (b) $2 \pi \gamma=3$; (c) $2 \pi \gamma=-3$; (d) $2 \pi \gamma=7.5$; (e) $2 \pi \gamma=-7.5$.

(v) Update the velocity distribution upstream using equations (2.31) and (2.32) and return to (ii) until the velocity magnitude along the whole free surface has converged.

\section{Results and discussion.}

The formulation of the problem and its solution procedure developed in section 2 makes it possible to consider a smooth body of an arbitrary shape as well as a body with a sharp corner, such as hydrofoil with a sharp trailing edge. For the former we shall use a circular cylinder for case study. Its radius $R$ is chosen as the characteristic length in the definition of the Froude number. We shall discuss below the features of the flow for different Froude numbers and depths of submergence.

\subsection{Flow past a submerged circular cylinder without gravity.}

The solution procedure is applied to solve the free surface flow past a circular cylinder beneath the free surface without the effect of gravity. In such a case $F=\infty$ and equation (2.3) can be simplified as $v(\xi) \equiv 1$. As the magnitude of the velocity is known on the free surface, only integro-differential equation (2.30), together with nonlinear equations $(2.23)$ - (2.27) has to be solved to determine the function $\beta_{b}(\xi)$. In figure 2 are shown the streamline patterns at different circulation $\gamma$ due to a vortex located at the centre of the cylinder. Due to the symmetry of the cylinder, these lines are symmetric about $x=0$.

The zero streamline is the line that passes through the stagnation point. It therefore splits into two lines at point $B$, which merge into one at point $A$. These two stagnation 
points are clearly shown in the figure. We may investigate the behaviour of the free surface at infinity using equation (2.32) in which $\Im(d z / d \zeta)_{\zeta=\xi+\pi \tau / 4}=d s / d \xi \sin \beta$

$$
y(\xi)=y_{D}+\int_{0}^{\xi} \sin \beta \frac{d s}{d \xi} d \xi, \quad \xi \rightarrow c
$$

where $\sin \beta=d y / d s$, and $s$ is arc length along the free surface. The behaviour of $\beta(\xi)$ on the free surface can be determined evaluating the leading order as $\xi \rightarrow c$. By taking into account equation $(2.23), \beta(\xi)_{\xi=c}=0$, from (2.35) for $\xi \rightarrow c$ we can obtain from the Taylor expansion

where

$$
\beta(\xi) \sim B_{1}(\xi-c), \quad \xi \rightarrow c
$$

$$
\begin{aligned}
B_{1} & =-\Im\left\{\frac{\vartheta_{1}^{\prime}(c-a+\pi \tau / 4)}{\vartheta_{1}(c-a+\pi \tau / 4)}+\frac{\vartheta_{1}^{\prime}(c-b+\pi \tau / 4)}{\vartheta_{1}(c-b+\pi \tau / 4)}-\frac{\vartheta_{4}^{\prime}(c-a+\pi \tau / 4)}{\vartheta_{4}(c-a+\pi \tau / 4)}-\frac{\vartheta_{4}^{\prime}(c-b+\pi \tau / 4)}{\vartheta_{4}(c-b+\pi \tau / 4)}\right\} \\
& -\Im\left\{\frac{1}{\pi} \int_{0}^{\pi} \frac{d \beta_{b}}{d \xi^{\prime}}\left[\vartheta_{1}^{\prime}\left(c-\xi^{\prime}+\pi \tau / 4\right)-\vartheta_{4}^{\prime}\left(c-\xi^{\prime}+\pi \tau / 4\right)\right] d \xi^{\prime}\right\}
\end{aligned}
$$

and dash on the Theta-function means the derivative. The leading order of the derivative $d s / d \xi$ is obtained form (2.37) taking into account that the function $\vartheta_{1}(\xi-c)$ along the free surface has a simple zero at $\xi=c$

$$
\frac{d s}{d \xi}=\frac{1}{v(\xi)}\left|K \frac{\vartheta_{4}^{2}(\xi-a+\pi \tau / 4) \vartheta_{4}^{2}(\xi-b+\pi \tau / 4)}{\vartheta_{1}^{2}(\xi-c) \vartheta_{4}^{2}(\xi-c)}\right| \sim \frac{K_{1}}{v(c) \vartheta_{1}^{2}(\xi-c)} \sim \frac{K_{1}}{(\xi-c)^{2}}, \quad \xi \rightarrow c
$$

where

$$
K_{1}=\left|K \frac{\vartheta_{4}^{2}(c-a+\pi \tau / 4) \vartheta_{4}^{2}(c-b+\pi \tau / 4)}{\theta_{4}^{2}(0)}\right| .
$$

By using (3.2) and (3.4), from (3.1) can be obtained

$$
y(\xi) \sim C_{1} \ln |\xi-c|, \quad \xi \rightarrow c,
$$

where $C_{1}=B_{1} / K_{1}$, and from (3.4)

$$
s(\xi) \sim-K_{1} /(\xi-c), \quad \xi \rightarrow c,
$$

By eliminating the parameter $\xi$ from (3.5) and (3.6), we obtain

$$
y(s) \sim-C_{1} \ln |s|, \quad|s| \rightarrow \infty .
$$

If the constant $C_{1}=B_{1} / K_{1} \neq 0$, the $y$-coordinate of the free surface tends to infinity for $|s| \rightarrow \infty$. However, the value of coefficient $B_{1}$ in (3.3) very much depend on the combination of the parameters $a, b, c$ determined from the system of equations (2.23), (2.25) and (2.27).

Streamline patterns for a submerged circular cylinder at the flowrate $Q=1$ for different circulations are shown in figure 2 . The free surface elevation in figure $2 a$ varies slowly in both directions away from the body, although it may not be obviously graphically visible. In figures $2 b$ and $2 d$ are shown streamlines for $\gamma=3 /(2 \pi)$ and $\gamma=7.5 /(2 \pi)$, respectively. The both stagnation points move towards the lower part of the cylinder, and the free surface elevation at infinity tends to $-\infty$. For the circulation $\gamma=-3 /(2 \pi)$ and $\gamma=-7.5 /(2 \pi)$, the stagnation points moves towards each other on the upper part of the cylinder, and the $y$-coordinate of the free surface tends to $+\infty$ at infinity. The flow patterns for larger flowrate $Q$ are shown in figure 3, which corresponds to large $y(x)_{x=0}$, or the distance between the free surface right above the body and its centre. As expected, 

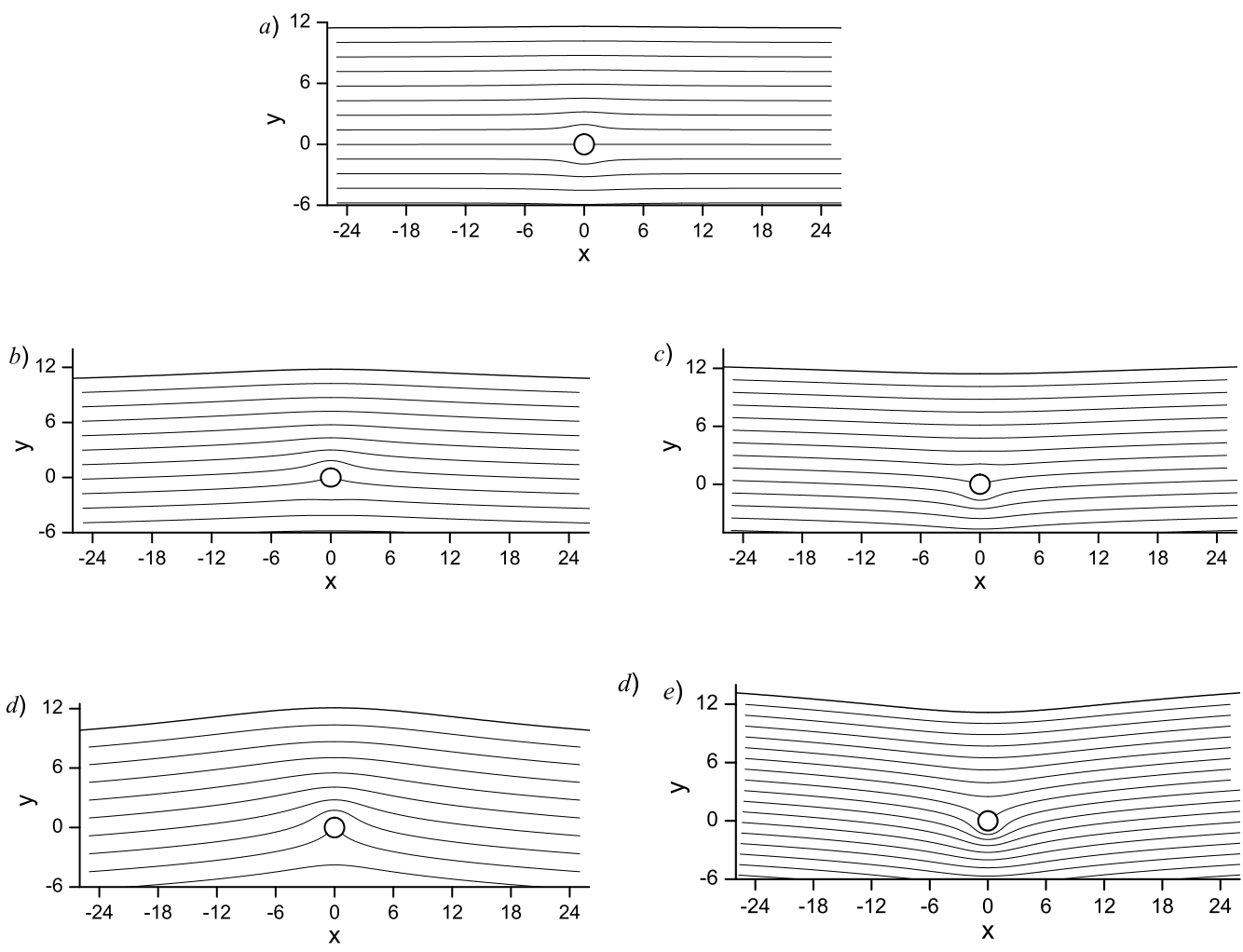

Figure 3. The streamline patterns for submerged circular cylinder at the flowrate $Q=10$ for different circulation: (a) $2 \pi \gamma=0$; (b) $2 \pi \gamma=3$; (c) $2 \pi \gamma=-3$; (d) $2 \pi \gamma=7.5$; (e) $2 \pi \gamma=-7.5$.

the effects of the body and circulation on the local free surface elevation become smaller than for the case shown in figure 2 .

The force acting on the body can be obtained from Blasius-Chaplygin theorem

$$
\bar{R}_{f}=X_{f}-i Y_{f}=\frac{i \rho U^{2} R}{2} \oint_{C_{b}}\left(\frac{d w}{d z}\right)^{2} d z=-\frac{i \rho U^{2} R}{2} \int_{0}^{\pi} \frac{d w}{d z} \frac{d w}{d \zeta} d \zeta
$$

where $C_{b}$ is a closed contour enclosing the body and the integration follows the positive (anti-clockwise) direction. The sign ${ }^{\prime}-^{\prime}$ in the last term appears due to the fact that integration route in the parameter plane from point $O_{-}$to $O_{+}$is in the opposite direction. Drag and lift coefficients are defined as follows

$$
C_{D}=\frac{2 X_{f}}{\rho U^{2} R}, \quad C_{L}=\frac{2 Y_{f}}{\rho U^{2} R} .
$$

The dependences of the lift coefficient on circulation $2 \pi \gamma$ at different $Q$ are shown in figure 4 . For the large $Q$ corresponding to large $y(0)$, the lift coefficient $C_{L} / 2 \rightarrow-2 \pi \gamma$, which corresponds to the Kutta-Joukovskii theorem in the case without free surface. For smaller $Q$ corresponding to smaller $y(0)$, the lift coefficient is smaller. Note, that the drag force is zero due to the symmetry of the flow about the $y$-axis.

\subsection{Flow past the submerged circular cylinder with gravity.}

For the case with gravity, we first reconsider those cases in Scullen \& Tuck (1995), and their results obtained from the numerical method will be used for comparison. 


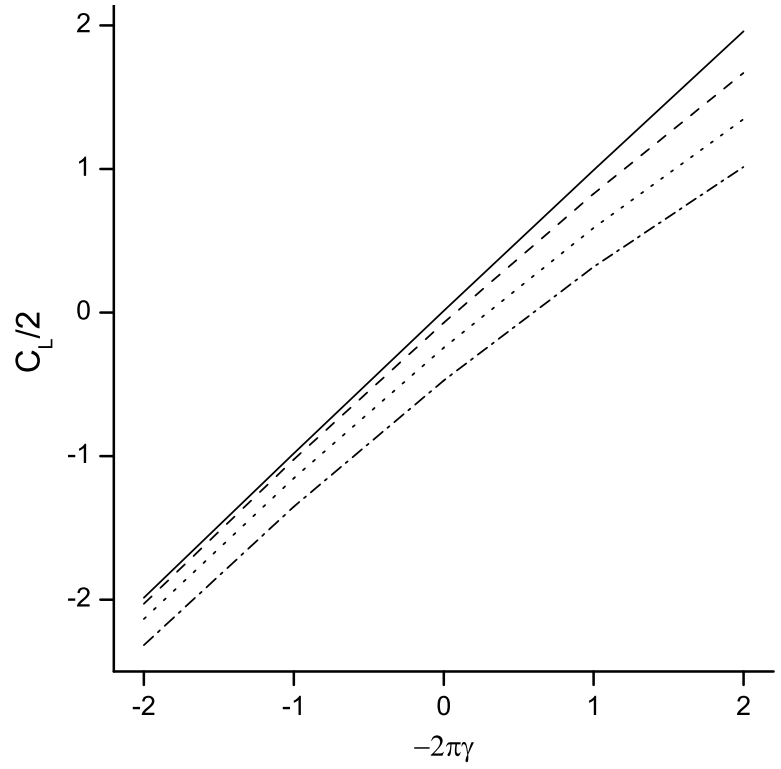

FiguRE 4. Lift force vs circulation for different flowrates between the body and the free surface: $Q=0.5$ (dash-dotted line), $Q=1.0$ (dotted line), $Q=2.0$ (dashed line), $Q=20$ (solid line).

The complete solution procedure presented in section 2.6 is used to predict the flow with gravity. For verification purposes we compare the obtained solution with Scullen \& Tuck (1995) results based on their numerical method. They represented the flow potential as a superposition of the potentials corresponding to the uniform flow, sources/sink and vortexes placed on the surfaces external to the fluid domain. The singularities due to sources were distributed at some distance over the free surface and within the circular cylinder close to its boundary. By using the dynamic and kinematic boundary conditions, the problem was reduced to a system of nonlinear equations solved by Newton method. Scullen \& Tuck (1995) presented results for various Froude numbers at depths of submergence $h \geqslant 4$.

In figure 5 are shown the free surface profiles for Froude number $F=2.75$ and the depth of submergence $h=7.55$, which gives $F_{h}=1$. They are compared with those of Scullen $\&$ Tuck (1995), shown by symbols. The circulation $\gamma_{0}=\Gamma_{0} /(2 \pi U R)=1 / F^{2}$ is the one at which the solution from the first approximation theory is waveless far downstream or $x \rightarrow \infty$, and it predicts zero drag force (Tuck \& Tulin (1992)). As can be seen from figure 5 , the present results are in an excellent agreement with those of Scullen \& Tuck (1995).

Further calculations are made for different submergences and Froude numbers. The free surface profiles are shown in figure 6 , in which the $x$-coordinate is scaled by $\lambda=2 \pi F^{2}$, the wavelength from the linear theory. The centre of the cylinder is shown by the solid circle. For Froude numbers $F=5$ and $F=3$ shown in figures $6 a$ and $6 b$, respectively, the wave amplitude initially increases when $h$ decreases, reaches a maximum value at a submergence $h=h_{m}$ and then decreases as the cylinder further approaches the free surface. For $h<h_{m}$ the free surface elevation above the cylinder is higher than the wave peak downstream. At very small depth the downstream wave almost disappears. Note, that for $h<1$, the top of the cylinder is above the still water level. Due to inertia of the incoming liquid, the free surface rises near the cylinder, and the liquid between the zero 


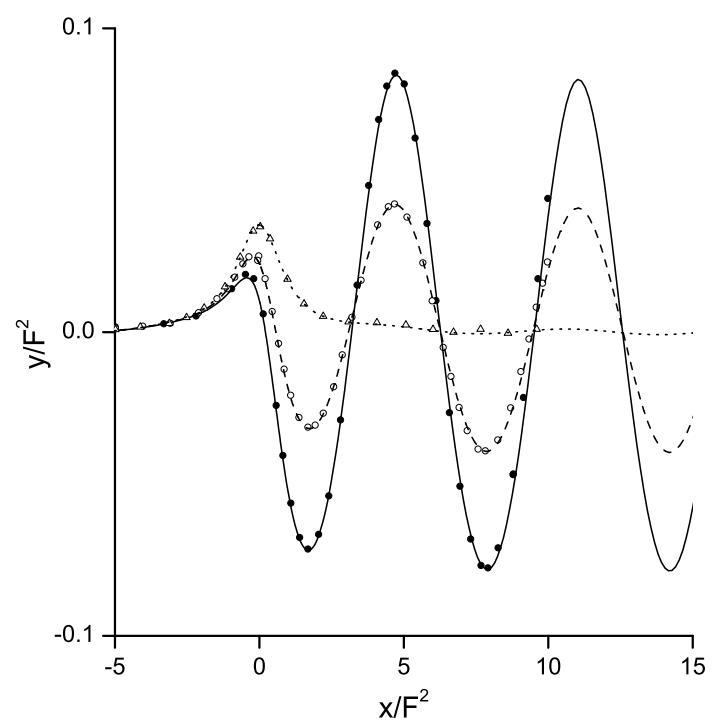

FiguRE 5. Free surface profiles for Froude number $F=2.75$ and the depth of submergence $h=7.55$, or $F_{h}=1$, at different circulations: $(a) \gamma=0$, (solid line), (b) $\gamma=0.5 \gamma_{0}$ (dashed line), (c) $\gamma=\gamma_{0}$ (dotted line). Symbols correspond to Scullen \& Tuck (1995) results and the lines to the present ones.
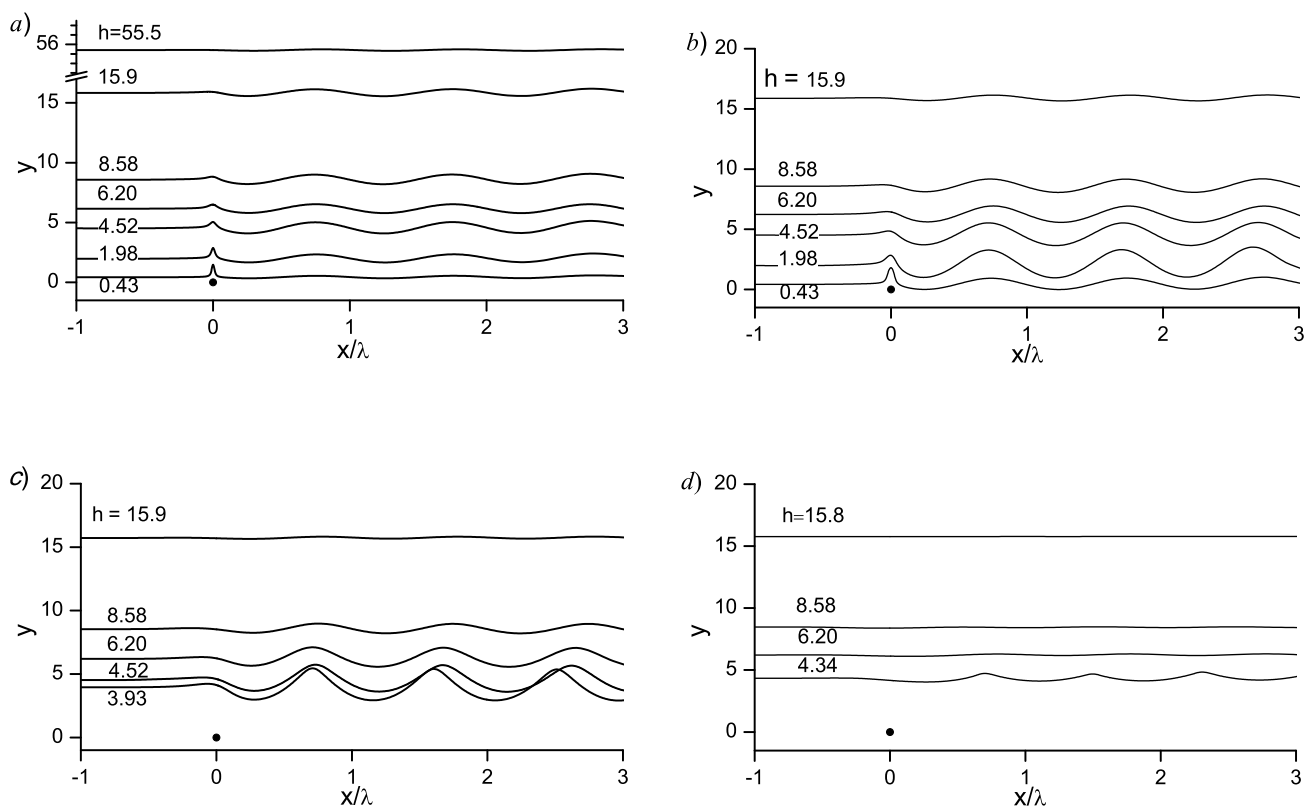

FiguRE 6. Free surface profiles for different depths of submergence and Froude numbers: $(a)$ $F=5,(b) F=3,(c) F=2,(d) F=1$.

streamline and the free surface passes the top of the cylinder. Then it moves down along the cylinder and merges with the main stream. Such flow may occur for those Froude numbers and submergences $h<1$, for which the free surface elevation $y-h<F^{2} / 2$, equivalent to the condition $v>0$ on the whole free surface.

For Froude number $F=2$ in figure $6 c$, the free surface is less disturbed than that in the 


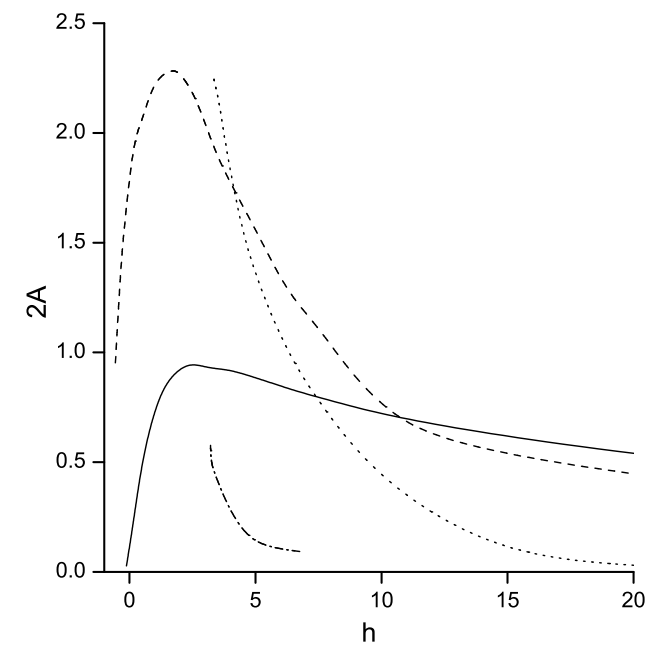

FiguRE 7. Wave height versus the depth of submergence for various Froude numbers: $F=5$ (solid line), $F=3$ (dashed line), $F=2$ (dotted line), $F=1$ (dot-dashed line).

case of larger Froude number when $h>15$. However, at smaller submergence, or $h<6.2$, the wave height becomes larger. The curve for $h=3.93$ corresponds to the smallest depth of submergence for which the converged solution could be obtained from the iteration process. For the velocity magnitude equal to 0.5 at the wave crest, the wave elevation therefore is $75 \%$ of the maximal possible elevation corresponding to $v=0$. As it can be seen, for the case of $h=3.93$, the wave crest becomes sharper than the wave trough, and wave elevation departs from the sinusoidal curve showing important nonlinear effect. As the wave height increases, and the wave length becomes smaller, which is consistent with nonlinear dispersion relationship (e.g, Lamb (1932), Art.250). As the submergence further decreases, the wave steepness becomes larger. However, the results shown are for the cases within limit for which non breaking waves can exist. Near or beyond the limit, the steady solution was not to be able to be found, which was also observed and discussed by Scullen \& Tuck (1995), Faltinsen \& Semenov (2008).

In our numerical method, when $h$ is nearer the critical value it becomes more difficult to obtain the solution, which is usually reflected by the non convergence during the iteration process. Convergence is obviously affected by the tolerance chosen for iteration. However, when the tolerance is sufficiently small, it no longer affects the limiting $h$ in the calculation. This was also observed and discussed by Haussling \& Coleman (1979); Scullen \& Tuck (1995) and by Faltinsen \& Semenov (2008). In these cases the wavelength and velocity continue to oscillate during the iterations, and the result does not converge.

For Froude $F=1$ in figure $6 d$, the free surface is weakly disturbed for $h>6.2$. For $4.34<h<6.2$ the wave slope grows rapidly and for $h<4.34$ steady wave patterns were not able to be obtained.

The downstream wave height, $h_{w}=y_{c r}-y_{t r}$ versus submergence is shown in figure 7 for Froude numbers $F=5,3,2$ and 1 . Here, $y_{c r}$ and $y_{t r}$ are the vertical coordinates of the wave crest and wave trough, respectively. For $F=5$ the maximal wave height is smaller than that for $F=3$. In the figure, at smaller Froude numbers $F=2$ and $F=1$, the wave height keeps increasing when the submergence decreases. Then, when the submergence further decreases, no converged solution could be obtained as discussed above. This is principally owing to a larger value $h_{w} / \lambda_{n}$, where $\lambda_{n}$ is the actual length of the nonlinear wave. In the cases of $F=5$ and 3 , the wave height $h_{w}$ first increases and 


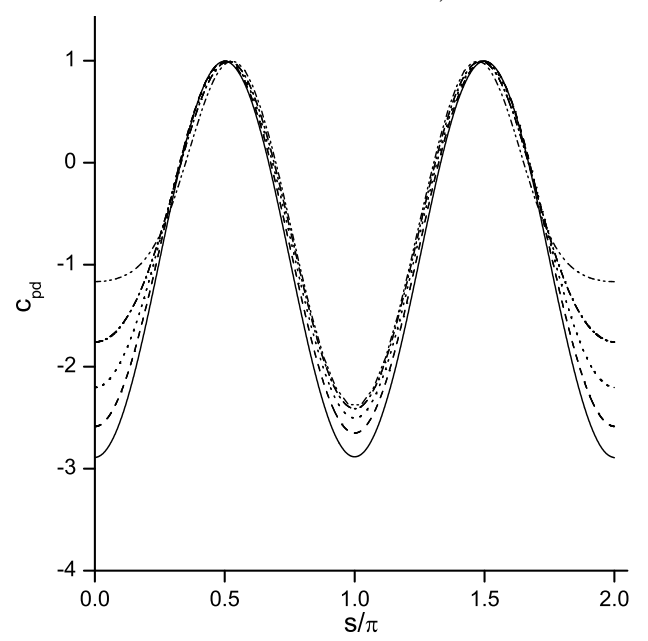

Figure 8. Pressure distribution along the cylinder for $F=5$ at different submergences: no free surface (solid line), $h=2.87$ (dashed line), $h=1.48$ (dotted line), $h=0.86$ (dash-dotted line) and $h=0.47$ (dash-dot-dot line).

then reaches a peak when submergence decreases. Beyond the peak $h_{w}$ decreases with $h$. Solution can still be obtained even $h<0$, for which the centre of the cylinder is already above the still water.

The pressure coefficient along the cylinder

$$
c_{p}=\frac{2\left(P-P_{\infty}\right)}{\rho U^{2}}=c_{p d}-\frac{2 y}{F^{2}}
$$

in which $c_{p d}$ is the dynamic pressure and the second term is the hydrostatic pressure. The dynamic pressure coefficient as the function of the arc length coordinate $s=S / R$ is shown in figure 8 for Froude number $F=5$. The values $s / \pi=0$ and $s / \pi=2$ correspond to the same top point of the cylinder, and $s / \pi=1$ corresponds to the bottom point. For the case without free surface, the pressure coefficients at the top and bottom points are the same, and they are the troughs of the curve. As the cylinder approaches the free surface, or $h$ decreases, the trough corresponding to the bottom point goes up slightly, and the one corresponding to the top point moves up much more. Therefore, excluding the buoyancy we can expect a downward vertical force whose magnitude increases as the cylinder approaches the free surface. For $h<1$, the top point of the cylinder is above the undisturbed free surface. The free surface upstream will rise near the cylinder and the pass over the top of the body similar to that shown in figure $2 a$. The peaks of the pressure coefficient $c_{p d}$ without the free surface in figure 8 correspond to the stagnation points, and the position is affected only slightly by the free surface.

Through the first approximation solution (Lamb (1932); Kochin, Kibel \& Roze (1964)) obtained the explicit equations for the resistance and lift force coefficients as

$$
\begin{aligned}
C_{D}^{\prime} h^{2}=\frac{X_{f}}{\pi \rho g R^{2}} h^{2}= & C_{D} \frac{h^{2} F^{2}}{2 \pi}=4 \pi\left(\frac{1}{F_{h}^{2}}-\gamma h\right) \exp ^{-2 / F_{h}^{2}} \\
C_{L}^{\prime} h^{2}=\frac{Y_{f}}{\pi \rho g R^{2}} h^{2}=C_{L} \frac{h^{2} F^{2}}{2 \pi} & =-\gamma F_{h}^{2} h\left(2 h^{2}+\gamma h-1\right)+4 \gamma h-\frac{1}{2} F_{h}^{2}-1-\frac{2}{F_{h}^{2}} \\
& +4\left(\frac{1}{F_{h}^{2}}-\gamma h\right)^{2} \exp ^{-2 / F_{h}^{2}} E i_{1}\left(\frac{2}{F_{h}^{2}}\right)
\end{aligned}
$$



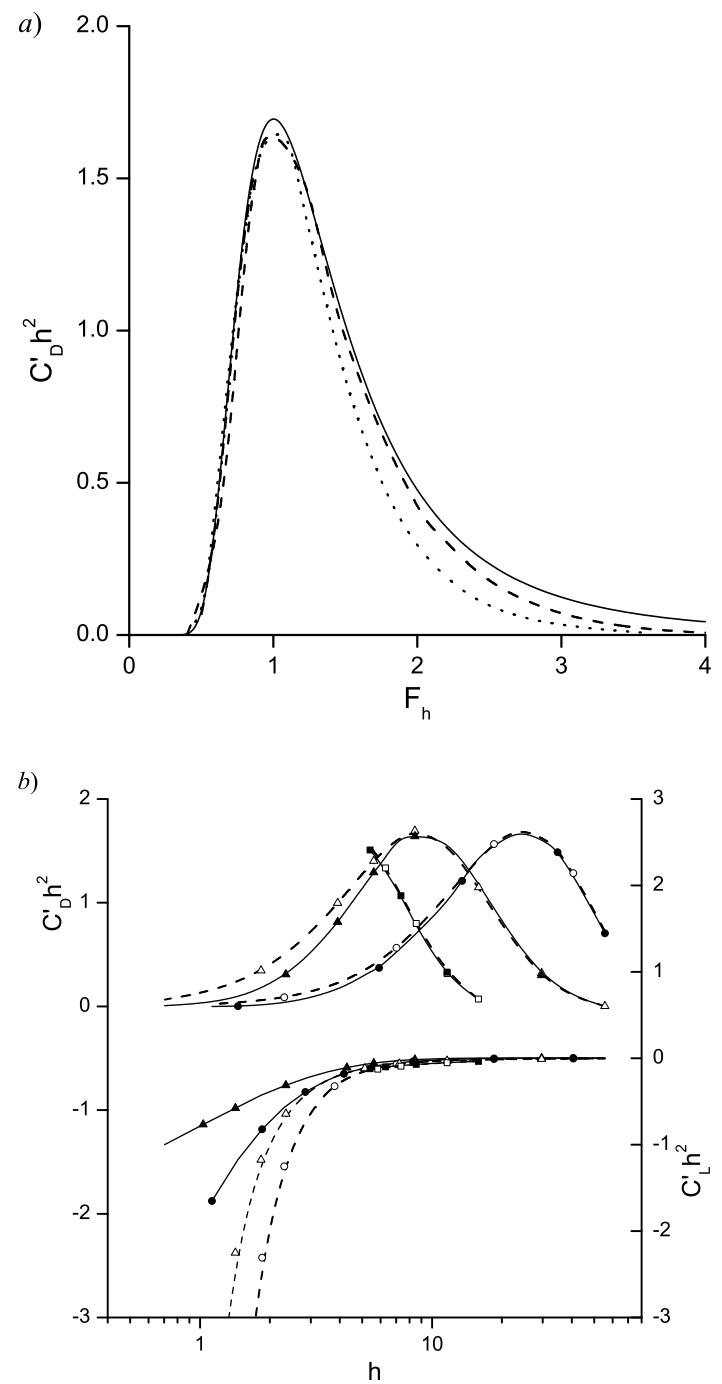

Figure 9. (a) Scaled drag and lift coefficients against $F_{h}=F / \sqrt{h}$ at different Froude number, $F$ : the first approximation theory (solid line) and the nonlinear theory for $F=5$ (dashed line), $F=3$ (dotted line) and $F=2$ (dash-dotted line, ends at $F_{h}=1.2$ ); $(b)$ scaled drag (upper curves) and lift (lower curves) coefficients as functions of the depth of submergence: nonlinear (solid lines and solid markers) and the first approximation theory (dashed lines and opened markers) for Froude $F=5(\bullet, \circ), F=3(\boldsymbol{\Delta}, \Delta)$ and $F=2(\boldsymbol{\square}, \square)$.

where $E i_{1}(z)$ is the exponential integral function. The coefficients $C_{D}^{\prime}$ and $C_{L}^{\prime}$ represent the force coefficients nondimensionalized by the buoyancy of the cylinder, $\pi \rho g R^{2}$. Results for the force at $\gamma=0$ are obtained from the present fully nonlinear theory and are given in figure $9 a$, in which $C^{\prime} h^{2}$ is plotted against submergence based Froude number, $F_{h}$, at different radius based $F$. For first approximation, at $\gamma=0$, the $C^{\prime} h^{2}$ in (3.11) depends on $F_{h}=F / \sqrt{h}$ only. It means that provided $F_{h}$ is the same $C^{\prime} h^{2}$ does not change with $F$. Thus results from the first approximation theory at $F=5,3$ and 2, (equation (3.11) are shown by the same solid line in $9 a$. However based on the present nonlinear solution, the results are very much affected by $F$ even at the same $F_{h}$. Nonlinear solution can be 
found for all the three $F$ when $F_{h}<1.2$, and the results are in a fairly good agreement with the first approximation solution. For $F_{h}>1.2$, no solution is obtained for the case of $F=2$. The results from $F=3$ and $F=5$, which correspond to a body of smaller radius relative to $h$, show some discrepancy with the first approximation solution. The difference is larger for the former, as the body is larger relative to the depth and therefore has a larger nonlinear effect.

The peaks of $C_{D}^{\prime} h^{2}$ in figure $9 a$ occur around the same $F_{h}$ at different $F$. The curves for $C_{D}^{\prime} h^{2}$ and $C_{L}^{\prime} h^{2}$ versus the depth of submergence are shown in figure $9 b$. The position is shifted to the left for large $F$ according to the relation $F_{h}=F / \sqrt{h}$. The normalized lift force coefficient, $C_{L}^{\prime} h^{2}$, becomes noticeable when $h$ becomes relatively small. The variation is faster for $F=5$ and then for $F=3$. For $F=2$ the curve ends at relative larger submergence.

\subsection{Flow past a submerged hydrofoil.}

The solution procedure derived in section 2 is also applicable for a body with a sharp corner point. In a such a case, Kutta-Joukovskii condition is to be imposed at the corner to ensure the velocity and the pressure are finite there. As a result, the circulation $\gamma$ should be obtained from such a condition, rather than being chosen randomly. Its chord length is chosen as the characteristic length $L$ in the following computation.

We consider hydrofoil NACA0012 which has the thickness of the trailing edge $2.5 \%$ of the hydrofoil thickness. In the present computations, we neglected the thickness of the trailing edge by taking it zero. The inner angle at the trailing edge is assumed as $\mu$. In order to satisfy the Kutta condition, the stagnation point $A$ and the trailing edge of the foil should coincide. With this assumption, the slope of the hydrofoil can be written as follows

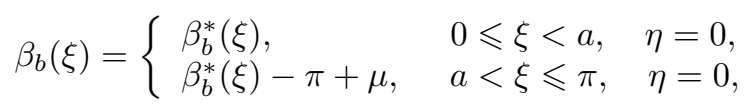

where $\beta_{b}^{*}(\xi)$ is a continuous function changed from 0 to $2 \pi-\mu$. By substituting (3.13) into (2.16) and evaluating the first integral over the step change in the function $\beta_{b}(\xi)$ at point $\xi=a$, we obtain the expression for the complex velocity for the hydrofoil

$$
\begin{aligned}
\frac{d w}{d z} & =v_{D}\left[\frac{\vartheta_{1}(\zeta-a)}{\vartheta_{4}(\zeta-a)}\right]^{\frac{\mu}{\pi}} \frac{\vartheta_{1}(\zeta-b)}{\vartheta_{4}(\zeta-b)} \exp \left[\frac{1}{\pi} \int_{0}^{\pi} \frac{d \beta_{b}^{*}}{d \xi} \ln \frac{\vartheta_{1}(\zeta-\xi)}{\vartheta_{4}(\zeta-\xi)} d \xi\right. \\
& \left.+\frac{i}{\pi} \int_{\pi}^{0} \frac{d \ln v}{d \xi} \ln \frac{\vartheta_{1}(\zeta-\xi-\pi \tau / 4)}{\vartheta_{4}(\zeta-\xi-\pi \tau / 4)} d \xi-i \beta_{O}\right]
\end{aligned}
$$

It is seen form the above equation that the complex velocity at the trailing edge has zero of order $\mu / \pi$. For the case $\mu=0$ which is assumed below, the first product in (3.14) disappears and velocity at the trailing edge becomes finite. Point $A$ will be still chosen at the trailing edge. However, this may not be a stagnation point anymore. The circulation $\gamma$ is obtained from (2.27). The parameters $a, b, c, K$ and $\tau$ are obtained from the system of equations $(2.23)-(2.26)$ and the equation

$$
s_{O A}=\int_{0}^{a} \frac{d s}{d \xi} d \xi
$$

where $d s / d \xi=|d z / d \zeta|_{\zeta=\xi}$, and $\xi=0$ corresponds to the point where the slope of the upper part of the foil is equal to zero. The system of integral equations from (2.30) to (2.38) has to be slightly modified by replacing the expression for the complex velocity (2.16) with (3.14).

The free-surface elevations for the flow past the NACA0012 hydrofoil at angle of 

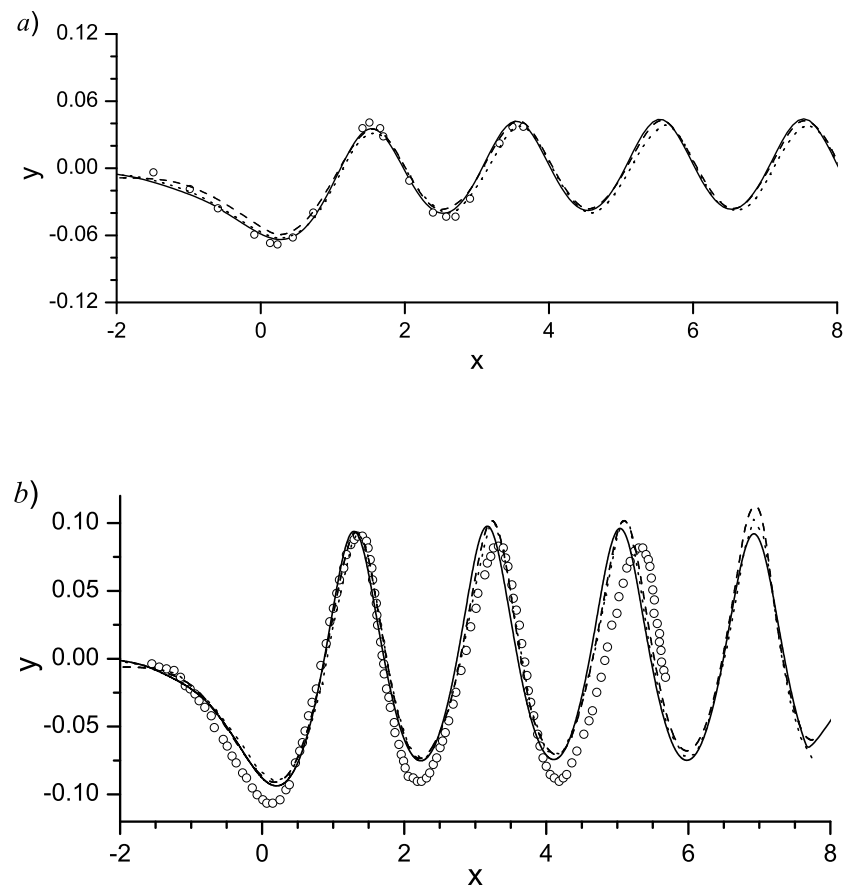

FiguRE 10. Free surface profiles past a NACA0012 hydrofoil at angle of attack $\alpha=5^{\circ}$, Froude number $F=0.567$ and depth of submergence $(a) h / L=1.256$ and $(b) h / L=1.0345$ measured from mid-chord point. Solid lines: present solution; dashed lines: Faltinsen \& Semenov (2008) analytical/numerical approach; dotted lines: Landrini et al. (1999) nonlinear numerical approach; symbol o: measurements from Duncan (1983).

attack $\alpha=5^{\circ}$ and Froude number based on the chord length of the foil $F=0.567$ are shown in figure 10 and compared with experimental data Duncan (1983) and with the results obtained through the fully nonlinear numerical approach by Landrini et al. (1999) and nonlinear solution by Faltisen and Semenov (2008). Agreement between the present nonlinear solution and that by Faltinsen \& Semenov (2008) as well as with that from the numerical simulation Landrini et al. (1999) is quite good. The agreement with the experimental data at larger submergence is also quite good. However there is some discrepancy between the experimental data and all the calculated data for the smaller depth of submergence $(10 b)$.

The dynamic pressure coefficient on the hydrofoil is plotted against $x$ for Froude number $F=2$ and angle of attack $\alpha=5^{\circ}$ is shown in figure 11. The origin is located in the middle point of the foil between the leading and trailing edges. The pressure coefficient with peak $c_{p d}=1$ at the stagnation point near the leading edge, $x=x_{B}$ gradually decreases along the lower side of the hydrofoil. On the upper side, the pressure coefficient rapidly drops to its minimal value and then gradually increases with $x$. The effect of submergence most pronounced on the upper side. It increases as the depth decreases, and even becomes larger than that on the lower side at the same $x$ on some part of the hydrofoil.

The effect of the submergence on the lift coefficient is shown in figure 12. The solid lines and symbols correspond to the lift coefficients obtained through the integration of the pressure around the hydrofoil, while the dashed lines and opened symbols are based on the Kutta-Joukowskii theorem $\left(Y_{f}=-\rho U \Gamma\right)$ for the unbounded flow domain, which 


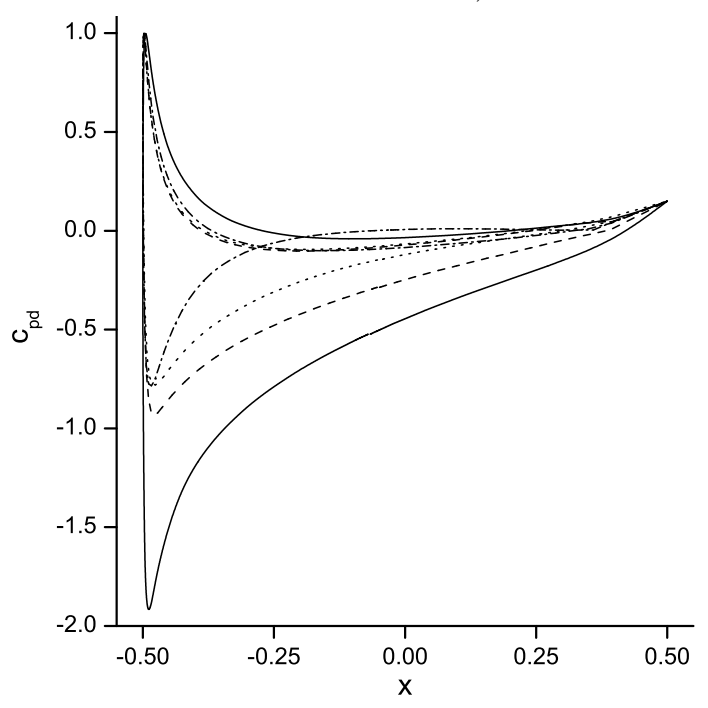

FigURE 11. Effect of submergence on the pressure distribution along the lower and upper sides of hydrofoil NACA0012 for Froude number $F=2$ and angle of attack $\alpha=5^{\circ}$ : no free surface (solid line), $h=0.74$ (dashed line), $h=0.33$ (dotted line) and $h=0.10$ (dash-dotted line).

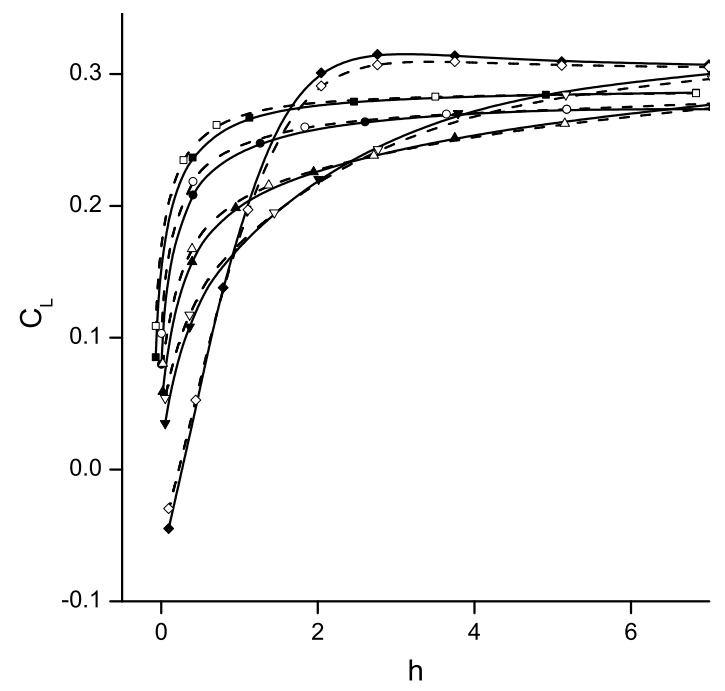

FiguRE 12. Effect of submergence on the lift coefficient for hydrofoil NACA0012 at the angle of attack $\alpha=5^{\circ}$ for Froude numbers $F=10,5,3,2$ and 1 (lines marked by squire, circle, triangle, reversed triangle and diamond, respectively). The solid lines and symbols correspond to integration of the pressure around hydrofoil, and the dashed lines and opened symbols correspond to the Kutta-Joukowskii theorem relating the lift force and circulation around the hydrofoil.

in the present notations is $C_{L}=2 \pi \gamma$, where $\gamma$ is obtained from equation (2.27). From the figure it is seen that the lift decreases as the hydrofoil approaches the free surface, which is consistent with the increase of the pressure on the upper side of the hydrofoil in figure 11. At small depths of submergence, the smaller the Froude number is, the smaller lift coefficient becomes, which may also become negative.

The wave resistance of a submerged body can be also determined by the wave energy 


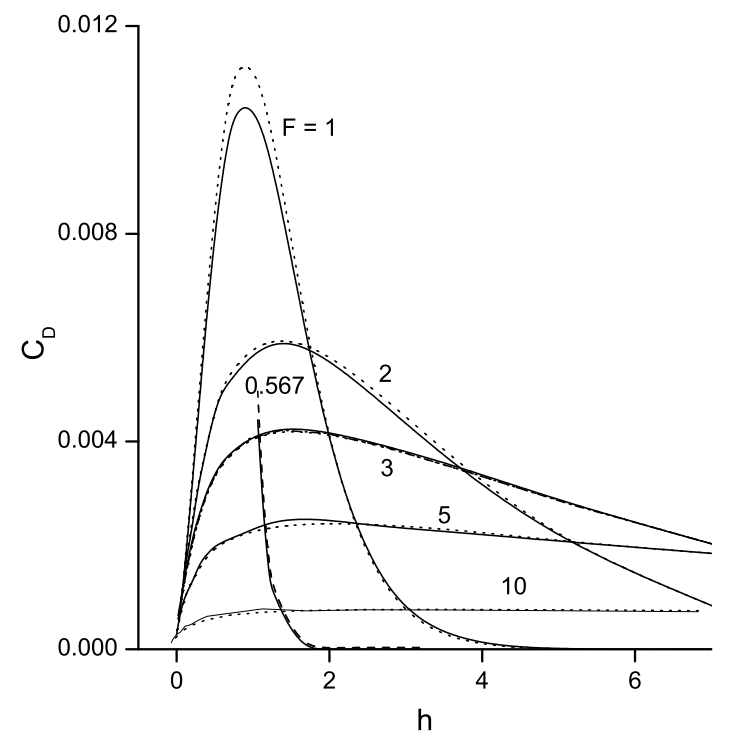

FIGURE 13. Effect of submergence on the wave resistance of the hydrofoil NACA0012 at the angle of attack $\alpha=5^{\circ}$ for Froude numbers $F=10,5,3,2,1$ and 0.567 . The solid and dashed lines correspond to equation (3.9) and(3.16), respectively.

flux downstream generated (Wehausen \& Liatone (1960)). For linear sinusoidal waves with height $h_{w}$, this may be simplified as (Newman (1977))

$$
C_{D}=\frac{h_{w}^{2}}{16 F^{2}}
$$

which does not depend on the body shape. In figure 13, the coefficients versus depth $h$ obtained using equation (3.8) and equation (3.16) are shown by solid and dashed lines, respectively. As the depth decreases, the wave height becomes larger and $C_{D}$ reaches its maximal value $C_{D \text { max }}$ at submergence $h_{m}$. For further decrease of the depth, $h<h_{m}$, the wave amplitude and the resistance, correspondingly, become smaller. For smaller Froude number the coefficient $C_{D \text { max }}$ becomes larger. However, at very small Froude number ( $F<1$ in the presented calculations), the maximal wave resistance cannot be reached due to wave breaking, that can be seen in figure (13) for $F=0.567$. Thus similar to a smooth body, the steady solution is not always possible for a hydrofoil either.

\section{Conclusions}

A fully nonlinear solution for the problem of steady free-surface gravity flow past a submerged body is presented in the form of analytical expressions for the complex velocity and derivative of the complex potential in a parameter plane. To deal with flow in the doubly connected domain, a cut is introduced, which does not have to be a streamline, as required in the previous work. Correspondingly a rectangular region in the parameter plane is used for the fluid domain, instead of the first quadrant which has been commonly adopted previously. Accordingly, the Theta-functions are used in the derived integral formulae for the complex velocity and derivative of the complex potential. They are in explicit form involving the magnitude of the velocity on the free surface and the velocity direction on the body surface, which are functions of the parameter variable 
varying along the horizontal sides of the rectangle. These functions are determined from a system of integral equations which are derived by enforcing the dynamic and kinematic boundary conditions. These integral equations are solved numerically using an iteration procedure.

The flows past a submerged circular cylinder without and with gravity are investigated by the presented formulation. In the former, the free surface elevation away from the body may tend to infinity logarithmically under some conditions. In the latter, at each given submergence $h$ below a critical value, the steady solution may not be obtained when the Froude number $F$ is within a certain range. This range increases when the submergence decreases. At a given Froude number, the steady solution may not exist when submergence is below a critical value. When the solution always exists at any $h$ at certain $F$, there is value $h=h_{m}$ at which the free surface wave height reaches its maximal value. As $h$ further decreases, the wave height will decrease. At very small $h$, the free surface becomes almost flat. The calculated drag and lift force coefficients are compared with those from the first approximation theory. For Froude number $F<2$ the force coefficients predicted by the first approximation theory and the fully nonlinear theory agree well for all submergences when the nonlinear steady solution exists. For $F>3$ and small submergence, the force coefficients predicted by the nonlinear theory may be several times smaller than those predicted by the first approximation theory.

The formulation is extended to a body with a shape corner, in which circulation is obtained from the Kutta condition at the corner. NACA0012 hydrofoil is used for the case study. The results obtained agree with experimental data, and those from other computational methods. It is shown that similar to the smooth body, the steady solution may not exist within a range of $F$ at a given $h$.

\section{Appendix A. Theta-functions}

Four types of Theta functions and their properties (Whittaker \& Watson (1927)):

$$
\begin{aligned}
& \vartheta_{1}(\zeta)=2 \sum_{n=1}^{\infty}(-1)^{n-1} q^{\frac{1}{4}(2 n-1)^{2}} \sin (2 n-1) \zeta, \\
& \vartheta_{2}(\zeta)=2 \sum_{n=1}^{\infty} q^{\frac{1}{4}(2 n-1)^{2}} \cos (2 n-1) \zeta, \\
& \vartheta_{3}(\zeta)=1+2 \sum_{n=1}^{\infty} q^{n^{2}} \cos 2 n \zeta, \\
& \vartheta_{4}(\zeta)=1+2 \sum_{n=1}^{\infty}(-1)^{n} q^{2 n} \cos 2 n \zeta,
\end{aligned}
$$

where $q=e^{\pi i \tau}$.

$$
\begin{array}{llrl}
\vartheta_{1}(\zeta+\pi) & =-\vartheta_{1}(\zeta), & \vartheta_{1}(\zeta+\pi \tau)=-q^{-1} e^{-2 i \zeta} \vartheta_{1}(\zeta), \\
\vartheta_{2}(\zeta+\pi)=-\vartheta_{2}(\zeta), & \vartheta_{2}(\zeta+\pi \tau)=q^{-1} e^{-2 i \zeta} \vartheta_{2}(\zeta), \\
\vartheta_{3}(\zeta+\pi)=\vartheta_{3}(\zeta), & \vartheta_{3}(\zeta+\pi \tau)=q^{-1} e^{-2 i \zeta} \vartheta_{3}(\zeta), \\
\vartheta_{4}(\zeta+\pi)=\vartheta_{4}(\zeta), & \vartheta_{4}(\zeta+\pi \tau)=-q^{-1} e^{-2 i \zeta} \vartheta_{4}(\zeta) .
\end{array}
$$

Theta functions can be expressed one through another as follows

$$
\vartheta_{1}(\zeta)=-\vartheta_{2}\left(\zeta+\frac{\pi}{2}\right)=-i P \vartheta_{3}\left(\zeta+\frac{\pi}{2}+\frac{\pi \tau}{2}\right)=-i P \vartheta_{4}\left(\zeta+\frac{\pi \tau}{2}\right)
$$




$$
\begin{aligned}
& \vartheta_{2}(\zeta)=\vartheta_{1}\left(\zeta+\frac{\pi}{2}\right)=P \vartheta_{3}\left(\zeta+\frac{\pi \tau}{2}\right)=P \vartheta_{4}\left(\zeta+\frac{\pi}{2}+\frac{\pi \tau}{2}\right) \\
& \left.\vartheta_{3}(\zeta)=P \vartheta_{2}\left(\zeta+\frac{\pi \tau}{2}\right)=P \vartheta_{1}\left(\zeta+\frac{\pi}{2}+\frac{\pi \tau}{2}\right)=\vartheta_{4}\left(\zeta+\frac{\pi}{2}\right)\right) \\
& \vartheta_{4}(\zeta)=i P \vartheta_{2}\left(\zeta+\frac{\pi}{2}+\frac{\pi \tau}{2}\right)=\vartheta_{3}\left(\zeta+\frac{\pi}{2}\right)=-i P \vartheta_{1}\left(\zeta+\frac{\pi \tau}{2}\right)
\end{aligned}
$$

Here, $P=q^{-1 / 4} e^{i \zeta}$.

This work is supported by Lloyd's Register Foundation (LRF) to which the authors are most grateful. LRF supports the advancement of engineering-related education, and funds research and development that enhances safety of life at sea, on land and in the air.

Declaration of Interests. None.

\section{REFERENCES}

Abramowitz, M. \& Stegun, I. A. 1964 Handbook of Mathematical Functions with Formulas, Graphs and Mathematical Tables. National Bureau of Standards Applied Mathematics, Washington.

Birkhoff, G. \& Zarantonello, E.H. 1957 Jets, wakes and cavities. New York, NY: Academic Press.

Chapman, J \& Vanden-Broeck, J.-M. 2006 Exponential asymptotics and gravity waves. J. Fluid Mech. 567, 299 - 326.

DaGan, G. 1971 Free-surface gravity flow past a submerged cylinder. J. Fluid Mech. 49(1), 179 $-192$.

DunCAN, J. 1983 The breaking and non-breaking wave resistance of a two-dimensional hydrofoil. J. Fluid Mech. 126, 507 - 520.

Dias, F. \& VAnden-Broeck, J.-M. 1989 Open channel flows with submerged obstructions. J. Fluid Mech. 206, $155-170$.

Dias, F. \& VAnden-Broeck, J.-M. 2004 Trapped waves between submerged obstacles. J. Fluid Mech. 509, $93-102$.

Faltinsen, O. M. \& Semenov, Y.A. 2008 The effect of gravity and cavitation on a hydrofoil near the free surface. J. Fluid Mech. 597, 371 - 394.

Forbes, L. K \& Schwartz, L. W. 1982 Free-surface flow over a semicircular obstruction. J. Fluid Mech., 114, $299-314$.

Gurevich, M. I. 1965 Theory of jets in ideal fluids. Academic Press, 585p.

Havelock, T. H. 1927 The method of images in some problems of surface waves. Proc. R. Soc. Lond. A 115, 268 - 280.

Havelock, T. H. 1936 The forces on a circular cylinder submerged in a uniform stream. Proc. R. Soc. Lond. A 157, $526-534$.

Haussling, H. J. \& Coleman, R. M. 1979 Nonlinear water waves generated by an accelerated circular cylinder. J. Fluid Mech. 92(4), $767-781$.

JoukovskiI, N.E. 1890 Modification of Kirchhof's method for determination of a fluid motion in two directions at a fixed velocity given on the unknown streamline. Matemat. sbornik XV (in Russian).

King, A. C. \& Bloor, M. I. G. 1989 A semi-inverse method for free-surface flow over a submerged body. Q. J. Mech. Appl. Maths 42, $183-202$.

Kiselev, O. M. \& Troepol'skaya, O. V. 1996 Translational motion of a cylinder below the free surface of a fluid. Fluid Dynamics 31(6), $802-813$.

Kochin, N.E. 1937 On the wave resistance and lift of bodies submerged in a fluid. Tsentral. Aero-Gidrodinam. Inst., Moscow. (in Russian) Translated in Soc. Naval Arch. Marine Engrs. Tech. \& Res. Bull. 1-8, 1951.

Kochin, N. E., Kibel, I. A. \& Roze, N. V. 1964 Theoretical Hydromechanics. 583p. John Wiley \& Sons, Ltd.

Lamb, H. 1932 Hydrodynamics. Cambridge University Press. 
LANDrini, M., Lugni, C. \& Bertram, V. 1999 Numerical simulation of the unsteady flow past a hydrofoil. Ship Technol. Res. 46, 14.

Michell, J.H. 1890 On the theory of free stream lines. Phil. Trans. Roy. Soc. London, Ser. A 181.

Milne-Thomson, L.M. 1968 Theoretical Hydrodynamics. 5th Edition, Dover Publications, New York.

Newman, J. N. 1977 Marine Hydrodynamics. The MIT Press. 448p.

SAlvesen, N. 1969 On higher-order wave theory for submerged two-dimensional bodies. J. Fluid Mech. 38, $415-432$.

Scullen, D. \& TuCK, E.O. 1995 Nonlinear free-surface flow computations for submerged cylinders. J. Ship Res. 39, $185-193$.

Semenov, Y. A. \& IAfrati, A. 2006 On the nonlinear water entry problem of asymmetric wedges. J. Fluid Mech. 547, $231-256$.

Semenov, Y. A. \& Wu, G. X. 2013The nonlinear problem of a gliding body with gravity. $J$. Fluid Mech. 727, $132-160$.

Terentiev, J.S., Kirschner, A.G. \& Uhlman, I.N. 2011 The Hydrodynamics of Cavitating Flows. Backbone Publishing Company.

Tuck, E.O. 1965 The effect of non-linearity at the free surface on the flow past a submerged cylinder. J. Fluid Mech. 22, $401-414$.

Tuck, E.O. \& Tulin, M.P. 1992 On satisfying the radiation condition in free-surface flows. 7th Int. Workshop on WaterWaves and Floating Bodies, Val de Reuil, France.

Vanden-Broeck, J.-M. 2010 Gravity-Capillary Free-Surface Flows. Cambridge, UK: Cambridge University Press.

Wehausen, J. V. 1973 The wave resistance of ships Advances in Applied Mechanics. Edited by Chia-Shun Yih, 13, $93-245$.

Wehausen, J. V. \& Liatone, E.V. 1960 Surface waves. Springer - Verlag, Berlin.

Whittaker, E. T. \& Watson, G. N. 1927 A Course of Modern Analysis. Cambridge University Press; 4th edition.

Wu, G.X. 1995 Radiation and difraction by a submerged sphere advancing in water waves of finite depth. Proc. R. Soc. Lond. A 448, $29-54$.

Wu, G.X. 1998 Wavemaking resistance on a submerged sphere in a channel. J. Ship Res. 42, 1 $-8$.

Wu, G.X. \& Eatock TAYLOR, R. 1988 Radiation and difraction of water waves by a submerged sphere at forward speed. Proc. R. Soc. Lond. A 417, $433-461$. 